\title{
A Non-Intrusive Geometrically Nonlinear Augmentation to Generic Linear Aeroelastic Models
}

\author{
Alvaro Cea ${ }^{\mathrm{a}}$, Rafael Palacios ${ }^{\mathrm{a}}$ \\ ${ }^{a}$ Department of Aeronautics, Imperial College London, United Kingdom
}

\begin{abstract}
A new approach to build geometrically-nonlinear dynamic aeroelastic models is proposed that only uses information typically available in linear aeroelastic analyses, namely a generic (linear) finite-element model and frequency-domain aerodynamic influence coefficient matrices (AICs). Good computational efficiency is achieved through a two-step process: Firstly, a geometric reduction of the structure is carried out through static or dynamic condensation on nodes along the main load paths of the vehicle. Secondly, manipulation of the resulting linear normal modes (LNMs), the condensed stiffness and mass matrices, and the nodal coordinates provides the modal coefficients of the intrinsic beam equations along these load paths. This preserves the LNMs of the original problem and augments them with the geometrically nonlinear terms of beam theory. The structural description is in material coordinates and modal AICs are thus naturally included as follower forces. Numerical examples include cantilever wings built using detailed models, for which effects such as nonlinear aeroelastic equilibrium, nonlinear dynamics and structural-driven limit-cycle oscillations are shown. Results demonstrate the ability of the methodology to seamlessly and efficiently incorporate critical nonlinear effects to (linear) arbitrarily large aeroelastic models of high aspect ratio wings.
\end{abstract}

Keywords: Dynamic aeroelasticity, high-aspect ratio wings, geometrically-nonlinear structures, reduced-order models,

Email address: r.palacios@imperial.ac.uk (Rafael Palacios) 


\section{Nomenclature}

$$
\begin{aligned}
& \boldsymbol{\eta}=\text { external load in modal coordinates } \\
& \boldsymbol{f}(s, t)=\text { beam internal forces in material coordinates } \\
& \boldsymbol{f}_{1}=\text { applied forces } / \text { moments per unit beam length } \\
& \Gamma_{1,2}=\text { nonlinear coupling coefficients in modal coordinates } \\
& \boldsymbol{h}(s, t)=\text { beam sectional angular momentum } \\
& \boldsymbol{\kappa}(s, t)=\text { beam sectional moment strains } \\
& K_{a}=\text { stiffness matrix after condensation } \\
& \boldsymbol{m}(s, t)=\text { beam internal moments in material coordinates } \\
& \boldsymbol{M}_{a}=\text { mass matrix after condensation } \\
& \boldsymbol{p}(s, t)=\text { beam sectional linear momentum } \\
& \phi_{1}(s)=\text { intrinsic velocity modes } \\
& \phi_{2}(s)=\text { intrinsic force modes } \\
& \boldsymbol{\psi}_{1}(s)=\text { intrinsic momentum modes } \\
& \boldsymbol{\psi}_{2}(s) \quad=\text { intrinsic strain modes } \\
& \boldsymbol{r}_{a}(s, t)=\text { beam position vector } \\
& \boldsymbol{q}_{1}(t)=\text { intrinsic modal coordinates (velocity component) } \\
& \boldsymbol{q}_{2}(t)=\text { intrinsic modal coordinates (stress resultant component) } \\
& \boldsymbol{R}_{a}(s, t)=\text { beam local coordinate transformation matrix } \\
& s \quad=\text { curvilinear coordinate along main load path } \\
& \boldsymbol{T}=\text { Transformation matrix in condensation process } \\
& t \quad=\text { time } \\
& \mathbf{v}(s, t)=\text { beam translational velocities in material coordinates } \\
& \boldsymbol{\omega}(s, t)=\text { beam angular velocities in material coordinates }
\end{aligned}
$$




$$
\begin{array}{ll}
\omega_{j} & =\text { natural angular frequency of mode } \mathrm{j} \\
U & =\text { free stream flow velocity } \\
\rho & =\text { free stream air density } \\
\boldsymbol{Q} & =\text { aerodynamic influence coefficient matrix } \\
\mathcal{A}_{s} & =\text { Roger's approximation matrices } \\
\boldsymbol{\lambda} & =\text { aerodynamic states }
\end{array}
$$

\section{Introduction}

Next-generation passenger aircraft will need to meet much stricter emissions and fuel efficiency targets. This will undoubtedly accelerate the push towards increased used of advanced lighter materials to reduce operating weight and configurations with very high aspect ratio wings to reduce induced drag. Aeroelastic effects will thus dominate many aspects of wing design, although higher flexibility may also have positive effects in damping some external disturbances, such as gusts. As a result, airframe design processes may need to be revised (Palacios et al., 2014), and, in particular, geometrical-nonlinear effects may need to be accounted for in stages of the analysis where they are currently neglected. This has already been the case in more radical configurations, such as large solar-powered aircraft (Noll et al., 2007), where linear analysis yielded non-conservative results as they missed critical nonlinear aeroelastic couplings (Patil and Hodges, 2004). While fully-coupled nonlinear aeroelastic simulation is now available (Qiao et al., 2018), certification of a new air vehicle currently requires 100,000s of load case simulations (Kier, 2017), as it considers maneuvers and gust loads at different velocities and altitudes, and for a range of mass cases and configurations. Statistical methods may help reduce the total simulation burden, yet there is still a need for a computationally-efficient models that provide an acceptable level of fidelity to describe the vehicle dynamics. Furthermore, design of control strategies also requires reduced models, 
and standard approaches with linear models can fail under large wing deformations (Wang et al., 2018).

Two main approaches have been considered so far to construct computationally-efficient aeroelastic models with geometrical nonlinearity, namely, descriptions based on beam theory, and model-order reduction methods. Geometrically nonlinear beams have been extensively used to study these problems (see, for instance, the review by Afonso et al. (2017)). While they have been very useful to understand key physics and explore the design space, they impose substantial limitations when detailed finite-element (FE) models are already available. Extraction of the equivalent beam properties is needed through either a homogenization process (Dizy et al., 2013) or a stick modelling approach (Riso et al., 2020). For large structures, it has been shown that either approach can lead to significant errors when compared to nonlinear simulations using built-up finite-element models (Palacios and Cea, 2019; Medeiros et al., 2020). Alternatively, system identification approaches have been proposed (Mignolet et al., 2013) that capture the nonlinear response of structure to construct reduced-order models (ROMs) for fast computations in nonlinear dynamic aeroelasticity (Medeiros et al., 2020). The main issue here lies in the prior calculation of a training database made of a large number of static nonlinear computations, which then acts as the model internal physics in the actual simulations. This approach also needs the full model to be suitable for nonlinear simulations, which could be a real constrain, e.g. on linear models calibrated against Ground Vibration Tests (GVT).

The goal of this work is to develop an efficient computational framework to study the geometrically nonlinear effects on already existing (linear) industrial-scale aeroelastic models, building on an alternative approach (Wang et al., 2015; Palacios and Cea, 2019) that does not require prior nonlinear computations on a full 3D finite-element model. Instead, the geometric layout of the structure and the coupling between modes in the intrinsic beam equations (Hodges, 2003) - that, by construction, take sectional velocities and internal forces as main 
variables - are employed. A feature of this approach is that mass and stiffness properties of the initial configuration require no updating as the model deforms. Furthermore, only quadratic terms of the main variables are needed to capture the complete space of geometric nonlinearities. As for the modelling of the aerodynamic forces, although 2-D airfoil unsteady aerodynamics are still often used for high-aspect-ratio wing aeroelasticity, 3-D effects have been shown to play an important role (Modaress-Aval et al., 2019) and will be considered here. The aerodynamic forces are obtained here from the Doublet-Lattice Method (DLM), which solves a linearization of the compressible, inviscid, unsteady flow equations in the 3-dimensional domain, and it is commonly used in linear aircraft aeroelastic analysis (Kim, 2019). It will be shown how key geometrically nonlinear effects (wing inextensionality, follower force effects), can be taken into account despite the linear - i.e. not updating with geometry - aerodynamic matrices obtained from the DLM. The Unsteady Vortex Lattice Method (UVLM) (Murua et al., 2012) is another potential method for geometrically-nonlinear aeroelastic simulations, as it fully accounts for large deformations both in the structure and the wake. However, it is substantially more computationally demanding and it is limited to incompressible conditions, therefore not appropriate for the typical flow regimes of commercial aircraft. The current description can also accommodate higher fidelity aerodynamic models, such as 3D panel methods (Kier, 2017) that include thickness effects, CFD-generated aerodynamic influence coefficient matrices (Güner et al., 2019), and it will be shown that it is suitable for an industrial loads and aeroelastics environment.

The structure of this paper is as follows. Sec. 2 presents the methodological approach proposed in this work; Sec. 3 shows static and dynamic aeroelastic results with three different models, all of which were originally built as linear MSC Nastran aeroelastic models; and Sec. 4 presents the main conclusions of the work. 


\section{Theoretical Formulation}

The starting point in the formulation is an arbitrarily complex FE model for which structural dynamic condensation techniques are employed to reduce the 3-dimensional components into nodes along all major load paths (a skeleton-like substructure). The resulting linear normal modes (LNMs) of the reduced structure are evaluated in intrinsic variables (velocities and strains) (Hodges, 2003) and used as a basis of a Galerkin-projection of the geometrically-nonlinear intrinsic beam equations in modal space (Palacios, 2017). We generalize previous formulations with a four field description (velocity, momentum, strain, and internal forces) that enables arbitrary reduction techniques and the generalization to structures with distributed inertia. The projected equations are solved in time domain with unsteady aerodynamic forces obtained from a doublet lattice.

\subsection{Structural model: nonlinear modal intrinsic theory}

The two-stage structural reduction method has been presented in detail in Palacios and Cea (2019) and is only summarized here. The emphasis will be in the integration of the nonlinear equations of motion with aerodynamic loading.

\subsubsection{Initial condensation approach}

Consider the solid domain $\Omega$, defining the 3-D structure, and a 1-D sub-domain in which the deformable curve $\Gamma$ defines an internal load path. The points conforming $\Gamma$ either belong to the 3-D structure or are a weighted average of neighboring points. After a finite-element discretization, the linear structural dynamics equations can be written as $\boldsymbol{M} \ddot{\boldsymbol{u}}_{n}+\boldsymbol{K} \boldsymbol{u}_{n}=\boldsymbol{F}$, with $\boldsymbol{M}$ and $\boldsymbol{K}$ the global mass and stiffness matrices, respectively; $\boldsymbol{F}$ the vector of nodal forces/moments and $\boldsymbol{u}_{n}$ the vector of nodal displacements/linear rotations, which include the nodes of $\Omega$ and $\Gamma$, as the two domains either share nodes or nodes in $\Gamma$ have been linked to $\Omega$ through interpolation elements. Their components are all given with respect to a common inertial reference frame. In order to obtain the condensed model, the degrees of 

described. A linear dependency is now assumed between the omitted and the active degrees

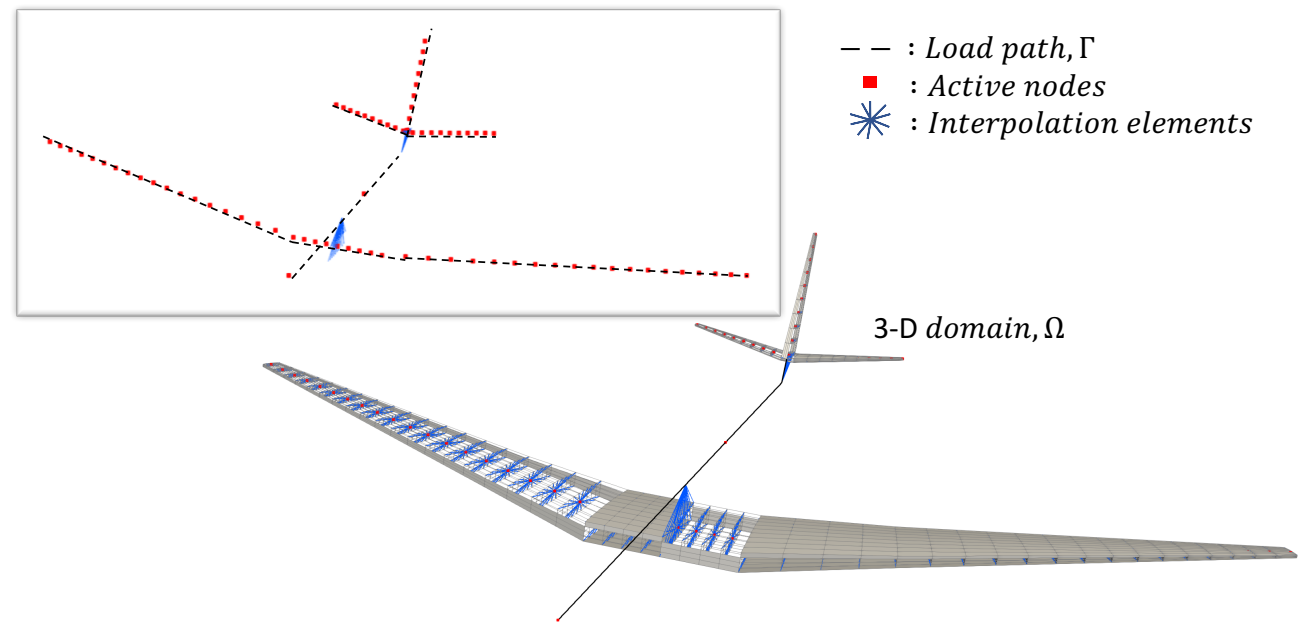

freedom (DoF) of the full model are divided into those on active (or master) nodes, located along the load path $\Gamma$, and those of the omitted nodes. Thus the FE matrices can be written as $\boldsymbol{K}=\left[\boldsymbol{K}_{a a} \boldsymbol{K}_{a o} ; \boldsymbol{K}_{o a} \boldsymbol{K}_{o o}\right]$ and $\boldsymbol{M}=\left[\begin{array}{lll}\boldsymbol{M}_{a a} & \boldsymbol{M}_{a o} ; \boldsymbol{M}_{o a} \boldsymbol{M}_{o o}\end{array}\right]$; the displacement vector reordered as, $\boldsymbol{u}_{n}=\left[\boldsymbol{u}_{a}^{\top} \boldsymbol{u}_{o}^{\top}\right]^{\top}$, and similarly for the LNMs of the unloaded structure $\boldsymbol{\Phi}=\left[\begin{array}{ll}\boldsymbol{\Phi}_{a}^{\top} & \boldsymbol{\Phi}_{o}^{\top}\end{array}\right]^{\top}$. Fig. 1 illustrates a typical definition of both 3-D and 1-D domains in a simplified aircraft structure. In the aerodynamic surfaces, the interpolation elements in blue relate the active nodes in the load path to the corresponding cross-sectional nodes of the 3-D structure. On the other hand, the fuselage is modelled as a rigid beam, and its nodes are part of both the full and reduced models. The nodes of the condensed model, in red, represent the discretization of $\Gamma$, where the geometrically nonlinear dynamics will be

Figure 1: Structural condensation, 3-D and 1-D domains (aircraft studied in (Palacios and Cea, 2019))

\footnotetext{
of freedom, $\boldsymbol{\Phi}_{o}=\boldsymbol{T}_{\text {oa }} \boldsymbol{\Phi}_{a}$, with $\boldsymbol{T}_{\text {oa }} \in \mathbb{R}^{o \times a}$ the transformation matrix between both sets. The quality of the transformation is determined by its accuracy to capture a subset of interest of the full-system LNMs. In general, the condensation is dependent on the frequencies and forms a nonlinear eigenvalue problem where each LNM, with natural frequency, $\omega_{j}$, has one
} 
transformation matrix,

$$
\boldsymbol{T}_{o a}\left(\omega_{j}\right)=\left(\boldsymbol{K}_{o o}-\omega_{j}^{2} \boldsymbol{M}_{o o}\right)^{-1}\left(\boldsymbol{K}_{o a}-\omega_{j}^{2} \boldsymbol{M}_{o a}\right) \approx-\left(\boldsymbol{K}_{o o}^{-1}+\omega_{j}^{2} \boldsymbol{K}_{o o}^{-1} \boldsymbol{M}_{o o} \boldsymbol{K}_{o o}^{-1}\right)\left(\boldsymbol{K}_{o a}-\omega_{j}^{2} \boldsymbol{M}_{o a}\right)
$$

This is the so called exact-condensation matrix, where we have also introduced Kidder's mode expansion (Qu, 2010) (right-hand-side expression in (1)) to avoid repeated calculation of the inverse in the first term. The first-order approximation of this equation is attained by letting $\omega_{j}=0$, thereby removing inertia effects. This results in a static condensation, also known as Guyan reduction (Guyan, 1965). Note that when the mass model consists only of lumped masses on the active degrees of freedom, $\boldsymbol{M}_{o o}=\boldsymbol{M}_{o a}=\mathbf{0}$, Guyan reduction is the exact condensation. After calculation of $\boldsymbol{T}_{o a}$, the transformation from the active set and the full model is defined as $T=\left[\begin{array}{ll}\boldsymbol{I}_{a} & T_{o a}^{\top}\end{array}\right]^{\top}$, with $\boldsymbol{I}_{a}$ the identity matrix of dimension a. The condensed stiffness and mass matrix in the restriction to the active set are obtained as $K_{a}=T^{\top} K T$ and $M_{a}=T^{\top} M T$, respectively. The LNMs in the active set are then $K_{a} \Phi_{a}=M_{a} \Lambda_{a} \Phi_{a}$, with $\Lambda_{a}$ the diagonal matrix of squared natural frequencies.

A first-order approximation including the effect of distributed inertia is obtained by selecting a single natural frequency in (1) for all LNMs. Therefore that one frequency is exactly approximated, while moving away from it loses accuracy. More accurate approaches have been developed using iterative methods. In particular, the iterative scheme shown in Ref. (Qu, 2010) based on Kidder expansion is considered here. The first step is to employ Guyan condensation to obtain $\boldsymbol{T}_{o a}^{(0)}, \boldsymbol{K}_{a}^{(0)}$ and $\boldsymbol{M}_{a}^{(0)}$. Let $\boldsymbol{\Phi}_{a}^{(0)}$ be the matrix that includes all the modes on the resulting condensed structure. Kidder expansion is then used to calculate the mode shapes at the omitted nodes, $\boldsymbol{\Phi}_{o j}^{(i)}=\boldsymbol{T}_{o a}\left(\omega_{j}^{(i-1)}\right) \boldsymbol{\Phi}_{a j}^{(i-1)}$, where the subindex $j$ refers to columns in the modal matrix. Next, the transformation matrix is recalculated, $\boldsymbol{T}_{o a}^{(i)}=\boldsymbol{\Phi}_{o}^{(i)}\left(\boldsymbol{\Phi}_{a}^{(i-1)}\right)^{-1}$, to finally yield the new $\boldsymbol{K}_{a}^{(i)}, \boldsymbol{M}_{a}^{(i)}$ and $\boldsymbol{\Phi}_{a}^{(i)}$. The level of fidelity preserved in the condensation will depend on how the master nodes are selected and 
on the ratio between master and omitted nodes. The resulting condensed matrices, $\boldsymbol{K}_{a}$ and $\boldsymbol{M}_{a}$, together with their LNMs, $\left(\boldsymbol{\Lambda}_{a}, \boldsymbol{\Phi}_{a}\right)$, and the spatial coordinates of the active nodes are the only information that is taken forward to the second stage, described next.

\subsubsection{Nonlinear intrinsic methodology}

The dynamics of a deformable curve along the load path, $\Gamma$, are described using Hodges' intrinsic beam formulation (Hodges, 2003). If $s$ is the curvilinear coordinate along $\Gamma$, we define $\mathbf{v}(s, t) \in \mathbb{R}^{3}$ and $\boldsymbol{\omega}(s, t) \in \mathbb{R}^{3}$ as the components in the local material frame of the inertial translational and rotational velocities, respectively. Their cross-sectional energy conjugates will be the linear and angular momentum, $\boldsymbol{p}(s, t) \in \mathbb{R}^{3}$ and $\boldsymbol{h}(s, t) \in \mathbb{R}^{3}$, respectively. Analogously, we define the sectional internal forces and moments in material coordinates, $\boldsymbol{f}(s, t) \in \mathbb{R}^{3}, \boldsymbol{m}(s, t) \in \mathbb{R}^{3}$, respectively, with their correspondent sectional force and moment strains, $\boldsymbol{\gamma}(s, t) \in \mathbb{R}^{3}$ and $\boldsymbol{\kappa}(s, t) \in \mathbb{R}^{3}$. The external forces and moments, $\boldsymbol{f}_{1}(s, t) \in \mathbb{R}^{6}$, are also given in the deformed material frame, i.e. they are follower forces, which is specially suitable for aerodynamic loading. The state variables are projected into the intrinsic form of the LMNs of the undeformed initial equilibrium equations. Using Einstein's summation convention, the modal projection is defined as,

$$
\begin{array}{rlrl}
\left(\mathbf{v}^{\top} \boldsymbol{\omega}^{\top}\right)^{\top} & =\boldsymbol{\phi}_{1 j}(s) q_{1 j}(t) & \left(\boldsymbol{p}^{\top} \boldsymbol{h}^{\top}\right)^{\top}=\boldsymbol{\psi}_{1 j}(s) q_{1 j}(t) \\
\left(\boldsymbol{f}^{\top} \boldsymbol{m}^{\top}\right)^{\top}=\boldsymbol{\phi}_{2 j}(s) q_{2 j}(t) & \left(\boldsymbol{\gamma}^{\top} \boldsymbol{\kappa}^{\top}\right)^{\top}=\boldsymbol{\psi}_{2 j}(s) q_{2 j}(t)
\end{array}
$$

where $q_{1 j}$ and $q_{2 j}$ are the dual intrinsic modal coordinates for the $j$-th LNM (Palacios, 2017), $\phi_{1}$ and $\psi_{1}$ are the respective velocities and momentum mode shapes, respectively, and $\phi_{2}$ and $\psi_{2}$ are the internal forces and strains intrinsic mode shapes. They are approximated here using the LNMs obtained from the condensation defined in section 2.1.1. For that purpose, we first define a continuous displacement/rotation mode shape $\boldsymbol{\phi}_{0}(s)=\mathcal{I}_{1}\left(\boldsymbol{\Phi}_{a}\right)$ 
obtained from linear interpolation in $\Gamma$ between the discrete values of $\boldsymbol{\Phi}_{a}$, where $\boldsymbol{\mathcal { I }}_{i}$ is the interpolation operator of order $i$. As shown by Palacios and Cea (2019), the velocity mode shape is obtained as $\phi_{1 j}(s)=\phi_{0 j}(s)$ (without rescaling for the frequencies). The momentum mode shape is analogously obtained from interpolation on the discrete velocity modes scaled by the condensed mass matrix $\boldsymbol{\psi}_{1 j}=\mathcal{I}_{0}\left(\boldsymbol{M}_{a} \boldsymbol{\Phi}_{a j}\right)$. The force components $\boldsymbol{\phi}_{2 j}$ are obtained from a summation-of-force approach from the internal nodal forces $\boldsymbol{K}_{a} \boldsymbol{\Phi}_{a j}$, and finally the strain modes $\boldsymbol{\psi}_{2 j}$ are obtained from spatial derivatives of the displacement modes along $\Gamma$.

The modal amplitudes are obtained from the nonlinear equations of motion after a Galerkin projection of the intrinsic beam equations, which we write in tensor notation as

$$
\begin{aligned}
& \dot{q}_{1 j}=\delta^{j i} \omega_{i} q_{2 i}-\Gamma_{1}^{j i k} q_{1 i} q_{1 k}-\Gamma_{2}^{j i k} q_{2 i} q_{2 k}+\eta_{j} \\
& \dot{q}_{2 j}=-\delta^{j i} \omega_{i} q_{1 i}+\Gamma_{2}^{i j k} q_{1 i} q_{2 k}
\end{aligned}
$$

where two third-order tensors introduce the gyroscopic, $\Gamma_{1}^{j k l}=\left\langle\boldsymbol{\phi}_{1 j}, \mathcal{L}_{1}\left(\boldsymbol{\phi}_{1 k}\right), \boldsymbol{\psi}_{1 l}\right\rangle$, and strainforce,$\Gamma_{2}^{j k l}=\left\langle\phi_{1 j}, \mathcal{L}_{2}\left(\phi_{2 k}\right), \boldsymbol{\psi}_{2 l}\right\rangle$ nonlinear modal couplings in the dynamic response. Two linear operators $\mathcal{L}_{1}(\phi)=[\tilde{\boldsymbol{v}} \mathbf{0} ; \tilde{\boldsymbol{u}} \tilde{\boldsymbol{v}}]$ and $\mathcal{L}_{2}(\phi)=[\mathbf{0} \tilde{\boldsymbol{u}} ; \tilde{\boldsymbol{u}} \tilde{\boldsymbol{v}}]$ are defined, for any $\boldsymbol{\phi}=\left(\boldsymbol{u}^{\top} \boldsymbol{v}^{\top}\right)$ and $\bullet$ the skew-symmetric operator. We have also used the inner product in the 1-D domain as $\langle\boldsymbol{\phi}, \boldsymbol{\psi}\rangle=\int_{\Gamma} \boldsymbol{\phi}^{\top} \boldsymbol{\psi} d s$, for any $\boldsymbol{\phi}, \boldsymbol{\psi} \in \mathbb{R}^{6}$. Finally, $\eta_{j}=\left\langle\boldsymbol{\phi}_{1 j}, \boldsymbol{f}_{1}\right\rangle$ is the modal projection of the external forcing terms and $\delta^{i j}$ the Kronecker delta. In this paper, we use superindices for matrix/tensor components and subindices for the elements of a column vector.

We will refer to equation (3) as the nonlinear modal reduced-order model (NMROM) of the original 3-D structure. It has dimension $2 \times N_{m}$, where $N_{m}$ is the number of LNMs retained from the condensed system in section 2.1.1. Local displacements and orientations can be recovered in postprocessing step either by time integration of local velocities, as it is done in rigid body dynamics, or through the integration of strains along the domain, as in the Frenet-Serret formulas of differential geometry. 


\subsection{Aeroelastic formulation}

The full aeroelastic system is formed by coupling the structural NMROM described above and the aerodynamic forces given by modal Aerodynamic Influence Coefficient matrices (AICs), in this case obtained from the DLM. In the aeroelastics literature the projected aerodynamic force coefficients on the mode shapes are often referred to as generalized aerodynamic forces, but we made no such distinction here. Since the structural equations are written in the material frame, the aerodynamic forces naturally appear as follower forces on the structure. Geometric changes in the aerodynamic grid as the structure deforms and their effect on wake geometry are however nonlinear effects that are not accounted for. Numerical examples in section 3 show that those geometric effects in the aerodynamic forces are minor on high-aspect ratio wings with moderate deflections (up to $20-25 \%$ of the wing semi-span). For higher deformations, the aerodynamic grid needs to be updated with the structural geometry, which can still be modeled usign the present methodology.

\subsubsection{Unsteady compressible aerodynamics}

The unsteady compressible aerodynamic equations are solved without viscosity and linearized around an initial velocity, $U$, associated to a reference free stream Mach number, Ma. The irrotationality condition of the flow allows the introduction of an acceleration potential that it is solved in the frequency domain with an approximation of the lifting surface using boxes with doublet lines (Rodden, 2011). The DLM computes the upwash at box $j$, due to a pressure in box $i$ is obtained as, $\bar{w}_{j}=A^{j i} \frac{2 \bar{p}_{i}}{\rho U^{2}}$, where the overbar refers to variables in frequency domain. The unsteady boundary conditions translate to non-penetration across the aerodynamic surfaces, which, in the absence of gusts, can be written in symbolic form as $\bar{w}_{j}=\left(U D_{1}^{j i}+i k D_{2}^{j i}\right) \bar{u}_{i}$, where $\bar{u}_{i}$ are the normal displacements at the aerodynamic grid points, $\boldsymbol{D}_{1}$ and $\boldsymbol{D}_{2}$ are the real and imaginary parts in the material differentiation matrix of the deflections to obtain the upwash, and $k$ the reduced frequency. Next, the normal 
forces $f_{j}$ at the aerodynamic grid points are obtained from integration of the pressure at each panel, $p_{i}$, that is, $\bar{f}_{j}=S^{j i} \bar{p}_{i}$.

The transformation of structural displacements onto the aerodynamic grid points is attained through an interpolation matrix, $\boldsymbol{G}$, whose transposed defines the consistent mapping from aerodynamic forces to the structural grid. Calculated forces are finally projected into the discrete LNMs to obtain a closed-form relation between generalized aerodynamic forces, $\overline{\boldsymbol{\eta}}$, and the modal displacements, $\overline{\boldsymbol{q}}_{0}$, as

$$
\overline{\boldsymbol{\eta}}=\frac{1}{2} \rho U^{2} \overline{\boldsymbol{Q}} \overline{\boldsymbol{q}}_{0}=\frac{1}{2} \rho U^{2}\left(\boldsymbol{\Phi}_{a}^{\top} \boldsymbol{G}^{\top} \boldsymbol{S} \boldsymbol{A}^{-1}\left(\boldsymbol{D}_{1}+i k \boldsymbol{D}_{2}\right) \boldsymbol{G} \boldsymbol{\Phi}_{a}\right) \overline{\boldsymbol{q}}_{0}
$$

where $\overline{\boldsymbol{Q}}$ are the AICs matrices. A rational-function approximation (RFA) is used to transform the frequency-domain physics into the time-domain. Despite a wide range of methods developed for decreasing the computational effort of the RFA (Karpel, 1982; Botez and Biskri, 2007; Dinu et al., 2005), classical methods, such as the one proposed by Roger (Roger, 1977), are often chosen for their robustness in a production engineering environment (Baker et al., 1999). Accordingly, $\boldsymbol{Q}$ is approximated here for a given Mach number as,

$$
\boldsymbol{Q}(k)=\mathcal{A}_{0}+(i k) \mathcal{A}_{1}+(i k)^{2} \mathcal{A}_{2}+\sum_{p=1}^{N_{p}} \frac{i k}{\gamma_{p}+i k} \mathcal{A}_{p+2}
$$

For a set of reduced frequencies and a given Mach number, $\overline{\boldsymbol{Q}}\left(k_{n}\right) \in \mathbb{R}^{N_{m} \times N_{m}}$ matrices are computed and the RFA is obtained through a least square fit. The last sum in Eq. (5) defines the aerodynamic states, $\bar{\lambda}_{p}=\frac{i k}{\gamma_{p}+i k} \overline{\boldsymbol{q}}_{0}$, where $\gamma_{p}$ is the aerodynamic lag associated to $\lambda_{p} \in \mathbb{R}^{N_{m}}$, for $p=1, \ldots, N_{p}$. The selection of these lags can greatly affect the quality of the aeroelastic simulations (Tiffany and Adams, 1988) and gradient-based optimization methods are often used to define them. In such case, the optimizer needs constraints to prevent the lags from collapsing into a single value, or a multipole approach as shown in (Eversman and 
Tewari, 1991). In this work, the optimization is carried out in a two-step process: firstly, a non-gradient discrete optimization is performed on the aerodynamic lags; the results are then the initial values of a gradient optimization. The error $\epsilon_{a}=\frac{1}{n} \sum \frac{\boldsymbol{\epsilon}\left(\overline{\boldsymbol{Q}}\left(k_{j}\right)-\boldsymbol{Q}\left(k_{j}, \boldsymbol{\gamma}_{p}\right)\right)}{\boldsymbol{\epsilon}\left(\overline{\boldsymbol{Q}}\left(k_{j}\right)\right)}$ yields an error metric for the optimization and the operator $\epsilon$ can be set as a weighted $l^{2}$ norm with higher relevance on the steady terms.

\subsubsection{Time-domain integration of aeroelastic system}

Applying the inverse Fourier transform both to equation (5) and to the definition of the aerodynamic states, the time-domain aerodynamic equations in nondimensional time, $t_{s}=\frac{U t}{c / 2}$, are recovered (with $c$ being a reference chord) as

$$
\begin{aligned}
& \boldsymbol{\eta}=\frac{1}{2} \rho U^{2}\left(\boldsymbol{A}_{0} \boldsymbol{q}_{0}+\frac{c}{2 U} \mathcal{A}_{1} \boldsymbol{q}_{1}+\left(\frac{c}{2 U}\right)^{2} \mathcal{A}_{2} \dot{\boldsymbol{q}}_{1}+\sum_{p=1}^{N_{p}} \boldsymbol{\mathcal { A }}_{p+2} \boldsymbol{\lambda}_{p}\right) \\
& \dot{\boldsymbol{\lambda}}_{p}=-\frac{2 U \gamma_{p}}{c} \boldsymbol{\lambda}_{p}+\boldsymbol{q}_{1} \quad \text { for } \quad p=1, \ldots, N_{p}
\end{aligned}
$$

where we note that both structural displacement and velocity modal amplitudes are used to define the inputs to the aerodynamic subproblem. Finally, substituting this expressions into equations (3) results in the aeroelastic NMROM equations of motion

$$
\begin{aligned}
\mathcal{M}^{i j} \dot{q}_{1 j} & =\delta^{i j} \omega_{j} q_{2 j}-\Gamma_{1}^{i j k} q_{1 j} q_{1 k}-\Gamma_{2}^{i j k} q_{2 j} q_{2 k}+\hat{\mathcal{A}}_{0}^{i j} q_{0 j}+\hat{\mathcal{A}}_{1}^{i j} q_{1 j}+\hat{\mathcal{A}}_{p+2}^{i j} \lambda_{p, j}+\eta_{1 i} \\
\dot{q}_{2 i} & =-\delta^{i j} \omega_{j} q_{1 j}+\Gamma_{2}^{j i k} q_{1 j} q_{2 k} \\
\dot{q}_{0 i} & =q_{1 i} \\
\dot{\lambda}_{p, i} & =q_{1 i}-\frac{2 U \gamma_{p}}{c} \lambda_{p, i}
\end{aligned}
$$

for $i, j, k=1, \ldots, N_{m}$ and $p=1, \ldots, N_{p}$. Here, we have introduced the constant matrix $\mathcal{M}^{i j}=\delta^{i j}-\frac{\rho c^{2}}{8} \mathcal{A}_{2}^{i j}$ and the re-scaled aerodynamic matrices $\hat{\mathcal{A}}_{0}^{i j}=\frac{\rho U^{2}}{2} \mathcal{A}_{0}^{i j}, \hat{\mathcal{A}}_{1}^{i j}=\frac{c \rho U}{4} \mathcal{A}_{1}^{i j}$, and $\hat{\mathcal{A}}_{2+p}^{i j}=\frac{\rho U^{2}}{2} \mathcal{A}_{2+p}^{i j}$. Finally, $\boldsymbol{\eta}_{1}$ refers to any external loads that do not come from the aerodynamics, projected into modal coordinates. The resulting aeroelastic description is 
therefore built with $2 N_{m}$ nonlinear differential equations that include geometrically-nonlinear modal couplings on the structure, $N_{m}$ integrators to evaluate the modal displacements and $N_{m} \times N_{p}$ linear ODEs for the aerodynamic states (due to our particular choice of RFA).

We can also define an aeroelastic state variable $\boldsymbol{x}=\left(\begin{array}{llllll}\boldsymbol{q}_{0}^{\top} & \boldsymbol{q}_{1}^{\top} & \boldsymbol{q}_{2}^{\top} & \boldsymbol{\lambda}_{1}^{\top} & \ldots & \boldsymbol{\lambda}_{N_{p}}^{\top}\end{array}\right)^{\top}$ and consequently rewrite Eqs. (7) in the nonlinear affine form

$$
\dot{\boldsymbol{x}}=\boldsymbol{g}(\boldsymbol{x}) \boldsymbol{x}+\hat{\boldsymbol{\eta}}
$$

where $\boldsymbol{g}(\boldsymbol{x})$ is a linear functional of the state variable. The aeroelastic system can be now linearized around any reference condition $\boldsymbol{x}^{*}$, to be $\boldsymbol{x}=\boldsymbol{x}^{*}+\Delta \boldsymbol{x}$, where typically the reference is a static equilibrium (i.e., $\boldsymbol{q}_{1}^{*}=\mathbf{0}$ and $\boldsymbol{\lambda}_{p}^{*}=\mathbf{0}$ for all $p$.). The linearized dynamic response follows

$$
\Delta \dot{\boldsymbol{x}}=\left[\boldsymbol{g}\left(\boldsymbol{x}^{*}\right)+\frac{\partial\left(\boldsymbol{g}(\boldsymbol{x}) \boldsymbol{x}^{*}\right)}{\partial \boldsymbol{x}}\right] \Delta \boldsymbol{x}+\Delta \hat{\boldsymbol{\eta}}
$$

Thus an efficient approach to aeroelastic analysis is generated whereby a steady-state nonlinear solution is firstly obtained, and subsequent stability analysis can be performed using the linear system described. When finite rotations need to be tracked, which applies whenever gravity or dead forces are considered, a parametrization using quaternions is used and additional states are included both in the full system and in its linearization (Wang et al., 2016). Finally, equations (7) can become very stiff if a relatively large number of modes is needed to achieve convergence. We note that high-frequency mode shapes may couple with lower frequency ones and affect the overall dynamics. A nonlinear residualization is therefore implemented where the equations above a selected cut-off frequency are timeaveraged, resulting in a set of differential-algebraic equations. 


\subsubsection{Implementation}

The formulation described above has been implemented into the software $\mathrm{FEM}_{4}$ INAS (Finite Element Model for Intrinsic Nonlinear Aeroelastic Simulations), written and parallelized in Python. It has been designed for applications in nonlinear aeroelasticity as a postprocess of linear aeroelastic models (in particular, MSC Nastran was used). The main workflow of the software is illustrated in Fig. 2 and is as follows: a) the structural process takes as inputs the full (linear) mass and stiffness matrices from an external FE solver and it computes the condensed matrices, the intrinsic modes and nonlinear modal coefficients; b) the aerodynamic process reads tabulated AICs (in particular, from DLM) and generates timedomain state-space model by employing an optimized RFA process. It is also possible to fully account for the geometric nonlinearities in the aerodynamics by periodically updating the AICs with the geometry as the structure deforms. c) The main solvers are inside a module that builds the required ODEs or DAEs of the aeroelastic NMROM with capability for static, dynamic and flight mechanics, follower and dead loadings, and multibody boundary conditions using Lagrange multipliers. Runge-Kutta or predictor-corrector integrators are used for time marching of the equations. d) recovery of displacement fields along the main load-paths is attained through integration of 1-D strains and velocities, as well as the full 3D state (if needed) using the transformation matrix $\boldsymbol{T}_{a o}$.

\section{Numerical Studies}

Three numerical studies are included to verify our implementation and demonstrate the suitability of the proposed approach to solve geometrically-nonlinear aeroelastic problems. Firstly, nonlinear vibrations are numerically investigated on a representative wing-box undergoing large displacements to explore the impact of different condensation techniques. The second example shows the nonlinear aeroelastic equilibrium and the dynamic response of a simple high aspect ratio wings, where results are compared to the literature. Lastly, a can- 


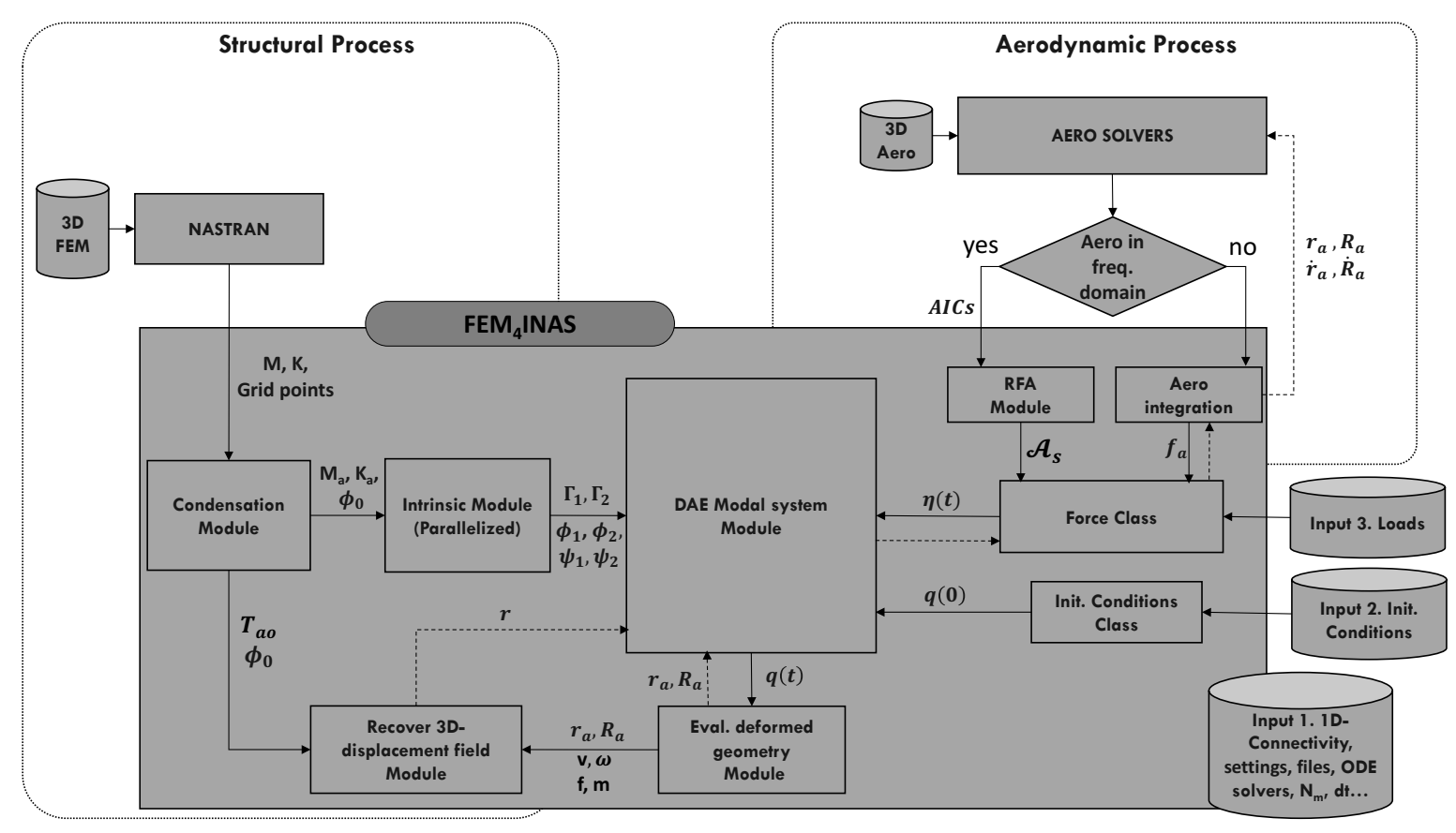

Figure 2: $\mathrm{FEM}_{4}$ INAS implementation

tilever wing built with shell, beam and lumped-mass elements is used to demonstrate a full nonlinear aeroelastic simulation.

\subsection{Representative wing structural models}

This test case demonstrates the suitability of the condensation methods described above for large FE models, and the accuracy of the NMROM approach for geometrically-nonlinear calculations. The initial design $\left(M_{1}\right)$, shown in Fig. 3(a), is a wing-box of $28.8 \mathrm{~m}$. semi-span, built to industrial practice and representative of current aircraft airliners. A total of 1462 elements are employed and the 1255 grid points are condensed to 23 active nodes in the center of the ribs. Composite material properties are used for the shells and mass is represented as continuous density of the structural elements. In order to depart from standard designs and increase its flexibility, a modified design $\left(M_{2}\right.$, shown in Fig. 3(b)) is considered in which the ribs are modified to have both holes and a $2 / 3$ reduction in thickness. In both wings a virtual load path is constructed through the center of each rib section using interpolation 
elements and the condensation nodes are shown in Fig. 3 in yellow. Figure 4 shows, for

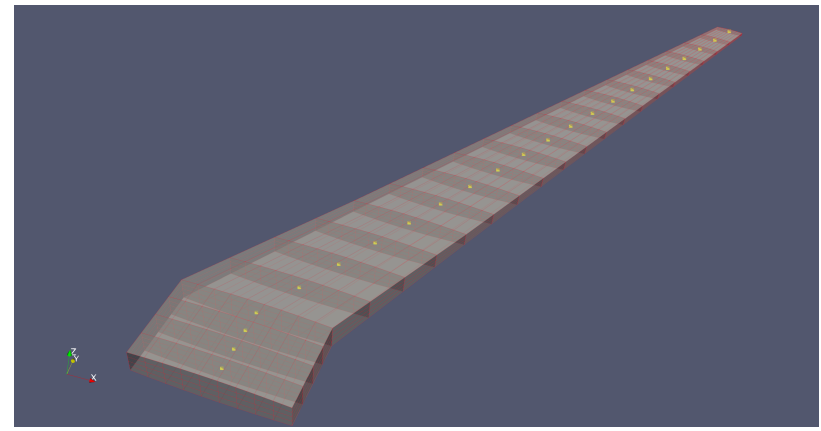

(a) Initial design $\left(M_{1}\right)$

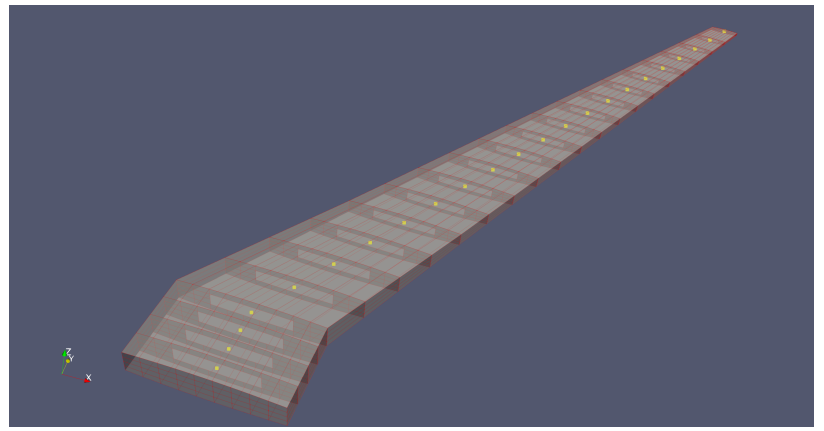

(b) Design with rib-holes $\left(M_{2}\right)$

Figure 3: Two representative wing models with the chordwise direction along the $\mathrm{x}$-axis and wing-span along the y-axis. Dots indicate the condensation nodes

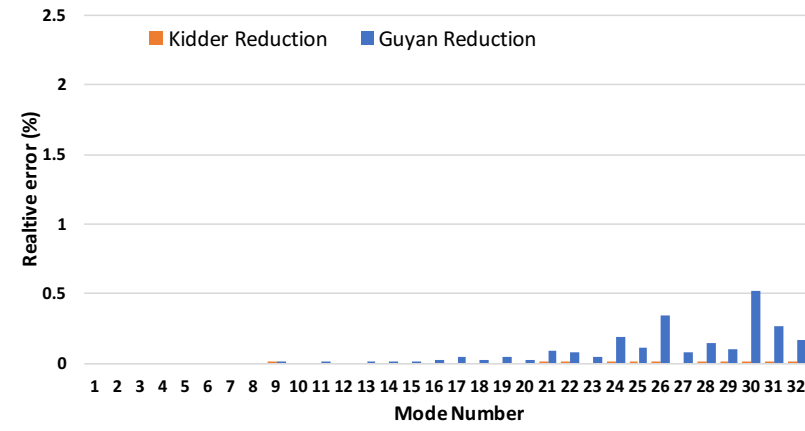

(a) $M_{1}$ wing

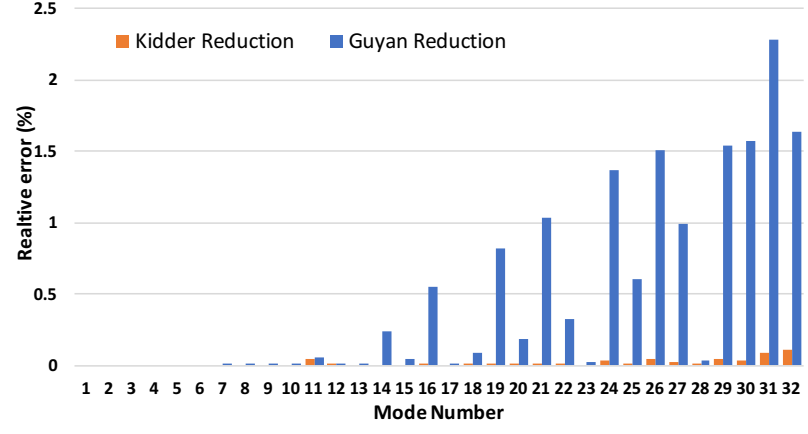

(b) $M_{2}$ wing

Figure 4: Relative errors in the models 
The density map of the stiffness matrices of the models gives some insight into the condensation process. Fig. 5 shows the condensed stiffness matrix for both the rigid $\left(M_{1}\right)$ and flexible $\left(M_{2}\right)$ wings, using Kidder condensation. For $M_{1}$ there are virtually no changes in the updated process of the Kidder reduction and this density map is identical to the one obtained from Guyan reduction. The density map of the wing with flexible ribs, Fig. 5(b), shows a much denser condensed matrix, which suggests that the iterative methods pack more information into the matrix to preserve the fidelity of the full model.

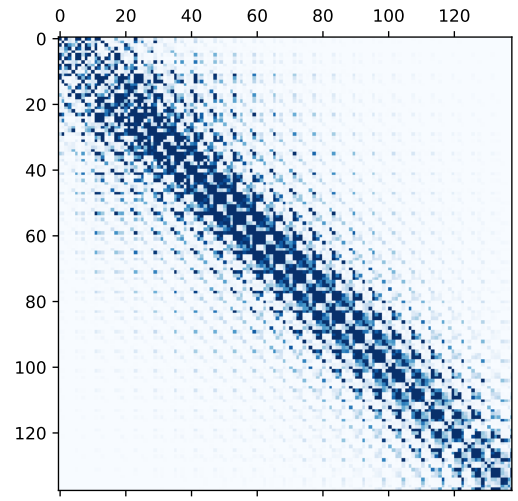

(a) $M_{1}$ wing

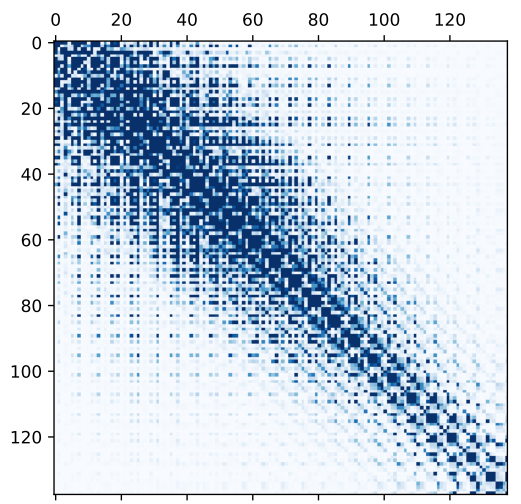

(b) $M_{2}$ wing

Figure 5: Density map of the condensed stiffness matrix in both wing models

Dynamic nonlinear simulations are carried out next and compared to MSC Nastran linear and nonlinear analysis (SOL 109 and 400, respectively) on the full FE model. A force is applied at the wing tip with a triangular loading profile given as

$$
f(a, b, c)= \begin{cases}a+\frac{1-a}{b} t & \text { if } t \leq b \\ 1-\frac{1}{b+c} t & \text { if } b<t \leq b+c \\ 0 & \text { otherwise }\end{cases}
$$

313 The follower force applied to the tip of $M_{1}$ is a ramp, $\boldsymbol{f}_{\text {tip }}=\left[-2 \times 10^{5}, 0,6 \times 10^{5}\right] f(0.05,4,0$. 

the wing semi-span. As expected, linear analysis overpredicts vertical displacements and

and the dynamic response is presented in Fig. 6, where results have been normalized with

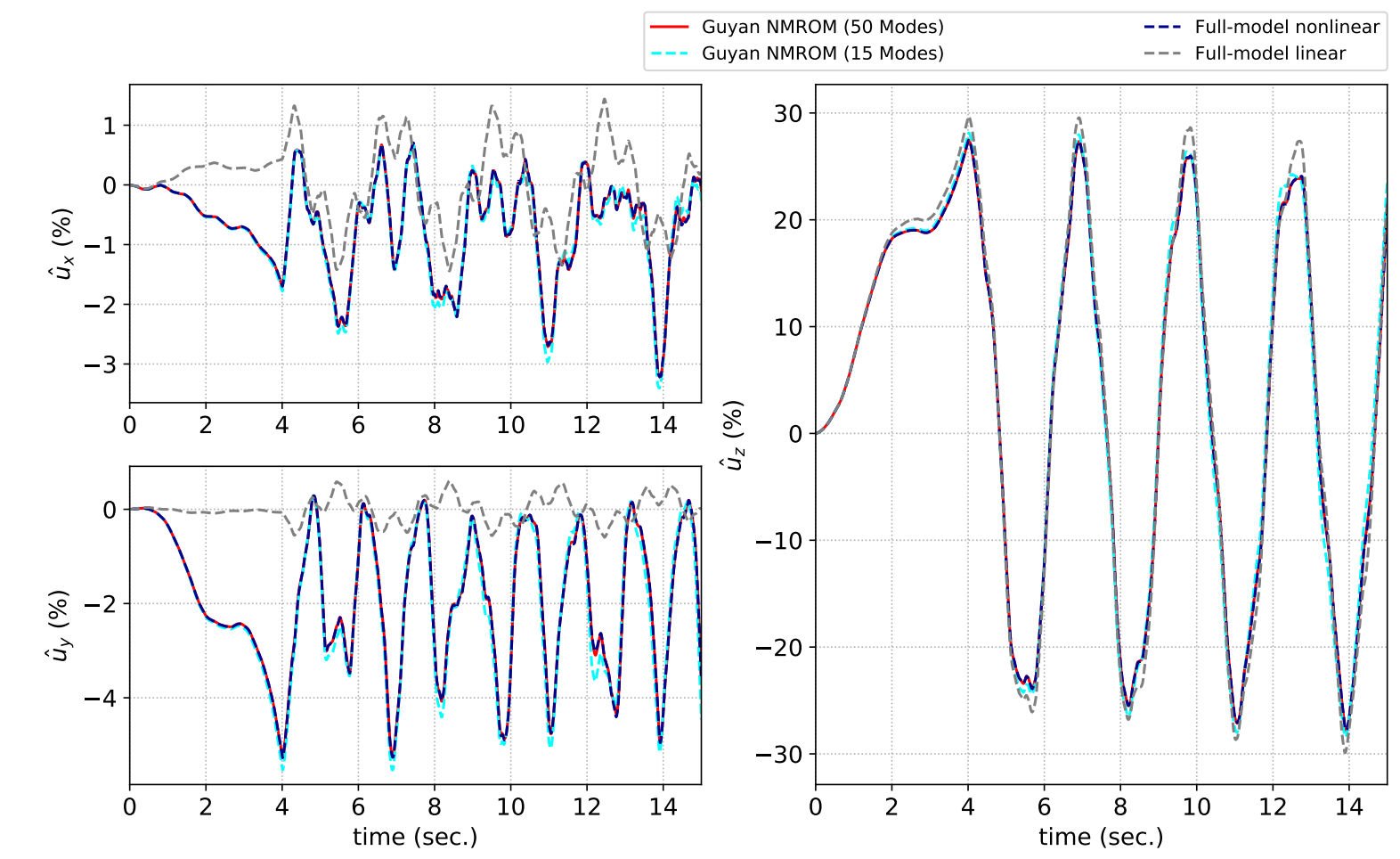

Figure 6: Span-normalized tip displacements in the dynamic simulation of $M_{1}$

does not capture displacements in the $x$ and $y$ directions. Using Guyan condensation, which is sufficient for this wing, NMROMs were built with 15 and 50 modes and run with timesteps of $6 \times 10^{-3}$ and $1.2 \times 10^{-3}$ respectively. The fully converged 50 modes NMROM and the nonlinear solution from the full FE solution are in complete agreement, even though deformations of $+28 \%$ of the semi span take place in the simulation. The accuracy of the NMROM with 15 modes is also good, yielding small differences with the full FE analysis and running at nearly real time (less than $30 \mathrm{~s}$ in an i7-6700 processor running at 3.40GHz), which is over 100 times faster than the time to run the full nonlinear simulation in MSC Nastran (3504 s in the same computer with a time step of $1.5 \times 10^{-3}$ for convergence). The NMROM model 50 modes needs $2226 \mathrm{~s}$ While this is still faster than the full FE, it highlights 
the relatively rapid growth in computational cost with the number of modes due to both the cost of evaluation of the nonlinear coupling tensors and the need for a smallest time step. For large modal basis, additional savings can be obtained using residualisation techniques, which reduced the computational effort by $75 \%$ in Palacios and Cea (2019). It is also worth remarking that the NMROM results come from a non-optimised Python implementation, while MSC Nastran is written and optimized for compiled language.

Next, the local angle of attack has also been measured along the wing. It is defined from the difference in vertical position of a central point and the corresponding leading edge. Fig. 7 shows the evolution in time of the angle of attack (AoA) in absolute values at the wing-tip for the simulation with design $M_{1}$. In these results, linear analysis overpredicts it by up to 3.6 degrees with respect to the full FE solution and the NMROM.

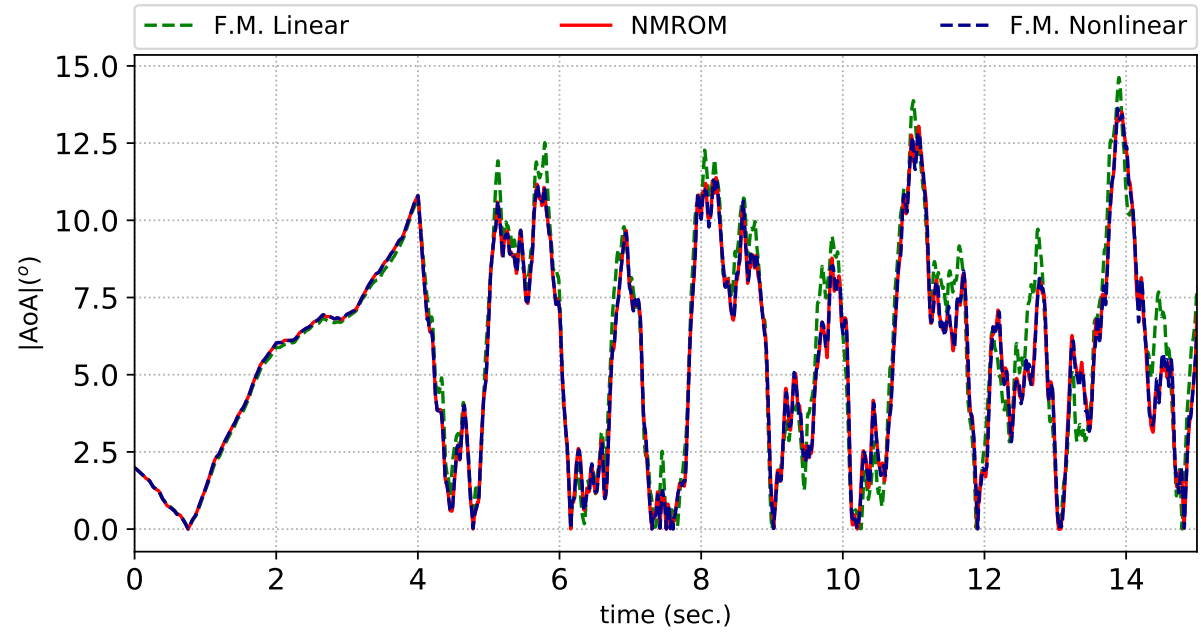

Figure 7: Angle of attack evolution in time at the wing-tip for design $M_{1}$

A similar exercise is run on the flexible design, $M_{2}$, now also adding moments in the $\mathrm{y}$-direction to excite higher frequencies. The forcing load at the tip is $f_{\text {tip }}=\left[3 \times 10^{5}, 0,1.5 \times\right.$ $\left.10^{5}, 0,6 \times 10^{5}, 0\right] f(0.05,3,0)$. Fig. 8 shows again the time histories of the three components of the wing tip displacements using the full-model, its linearization, and NMROM with 50 modes using both condensation schemes. Linear analysis shows even larger errors than in the 

largely driven by the dominant low frequency dynamics. The evolution of the error with

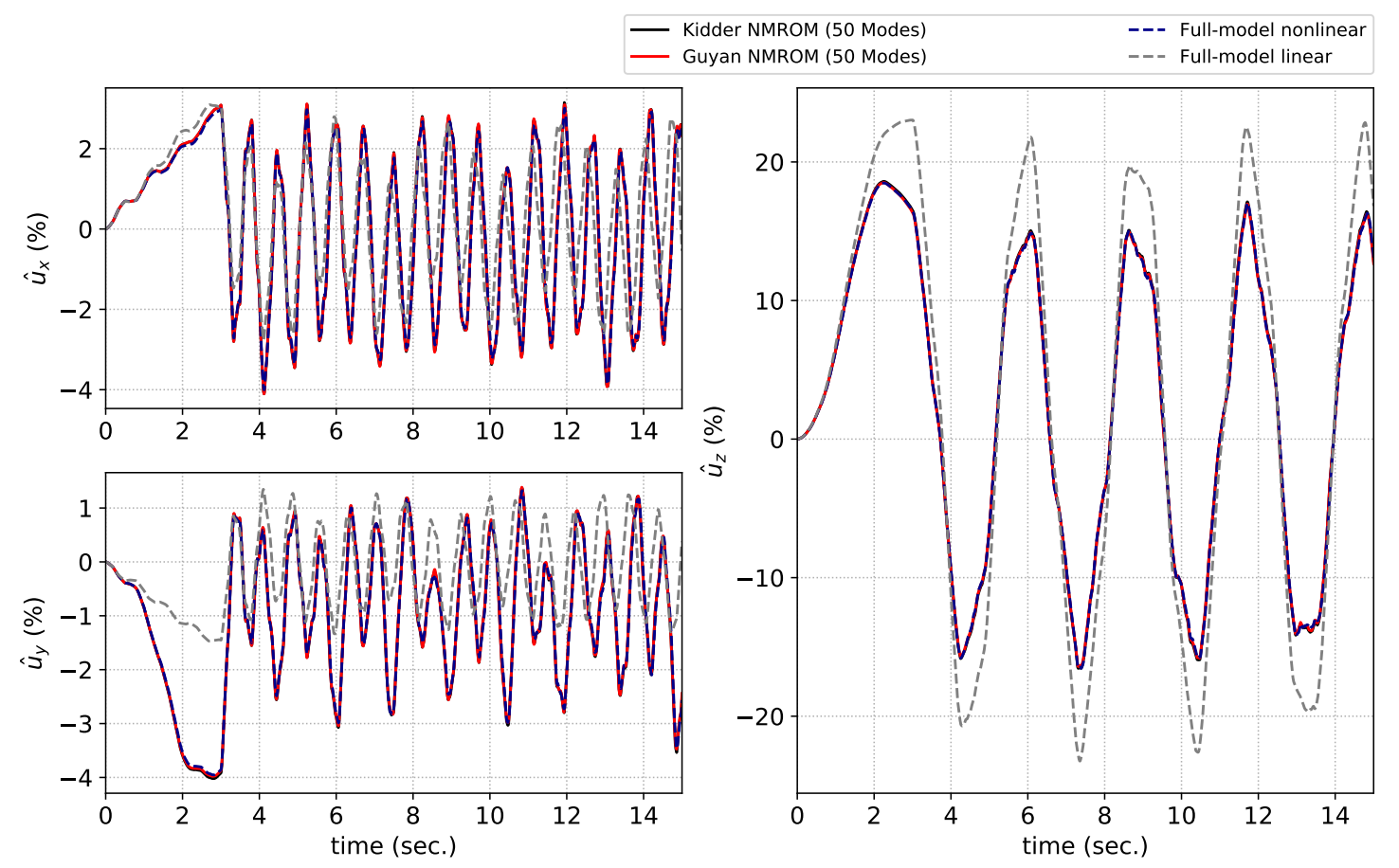

Figure 8: Span-normalized tip displacements in the dynamic simulation of $M_{2}$

previous example, and both condensation methods show again excellent comparison with the full FE model. This is the case even though the Kidder method was shown to have a small improvement in the higher modes, which were seen to be excited in this nonlinear simulation. These results suggest that the overall accuracy in the large-amplitude simulations is still

the number of modes in the solution is assessed by using a 2-norm of the tip displacements difference between the nonlinear solution on the full FE model and the NMROM averaged over time and it is shown in figure 9. An error below $1 \%$ is achieved with 15 LNMs, and we consider results sufficiently converged above 30 modes. For reference, the solution of $M_{2}$ took 298 s using 30 LNMs. Therefore, apart from the computational aspects underlined above, it is clear that the size of the modal basis makes the biggest difference in attaining both accurate and efficient calculations. 


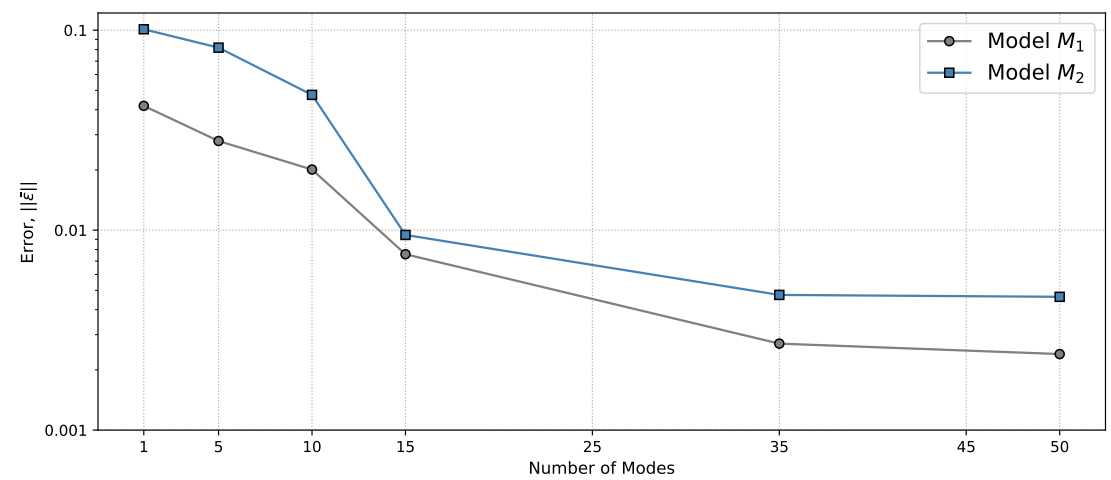

Figure 9: Error between NMROM and full model with number of modes in the NMROM solution

\subsection{Static and dynamic aeroelasticity of a highly flexible wing}

A very flexible wing model is built next to verify the implementation of the proposed nonlinear aeroelastic solver and assess the validity of the linear aerodynamic model. The wing considered is that of Patil et al. (2001), which has been extensively used in the literature as a validation test case for static aeroelasticity with geometrically-nonlinear models. As the original wing is dynamically unstable, the elastic axis has been displaced forward for the results in this paper, which uses the dynamic equations of motion to compute equilibrium conditions. The wing has a span of $16 \mathrm{~m}$ and chord of $1 \mathrm{~m}$, with no sweep or taper. The elastic axis is at $25 \%$ of the chord, the in-plane and out-of-plane bending stiffness are $6 \times 10^{6}$ $\mathrm{Nm}^{2}$ and $2 \cdot 10^{4} \mathrm{Nm}^{2}$, respectively, the torsional stiffness is $10^{4} \mathrm{Nm}^{2}$, the mass per unit length $0.75 \mathrm{~kg} / \mathrm{m}$ and the torsional inertia $0.1 \mathrm{Kg} \cdot \mathrm{m}$. It flies at $25 \mathrm{~m} / \mathrm{s}$ with air density 0.0029 $\mathrm{kg} / \mathrm{m}^{3}$.

For this test case, the structural model is based on beam elements in MSC Nastran and therefore no condensation is necessary. This simplification allows us to use SHARPy (del Carre et al., 2019) as the reference for comparison. SHARPy is a well-validated solver based on geometrically-nonlinear beams and unsteady vortex-lattice aerodynamics, which is the current state of the art for nonlinear dynamic aeroelastic simulation of high-aspect-ratio wing. For small deformations SHARPy and the aeroelastic NMROM are equivalent formulations, 
while for large amplitudes the only difference is the effect of change of wing geometry in the aerodynamic model in SHARPy, which is neglected in $\mathrm{FEM}_{4}$ INAS. This assumption, which gives huge computational savings, is therefore tested below. The wing is clamped at the root and the model has been created in both solvers with 16 beam elements and a $32 \times 5$ aerodynamic lattice. Firstly, the aeroelastic equilibrium conditions are determined for different angles of attack at the wing root. Fig. 10 presents the comparison between SHARPy and an aeroelastic NMROM simulation converged with 35 modes. Results are presented normalized with the wing semi-span, with and without gravity.

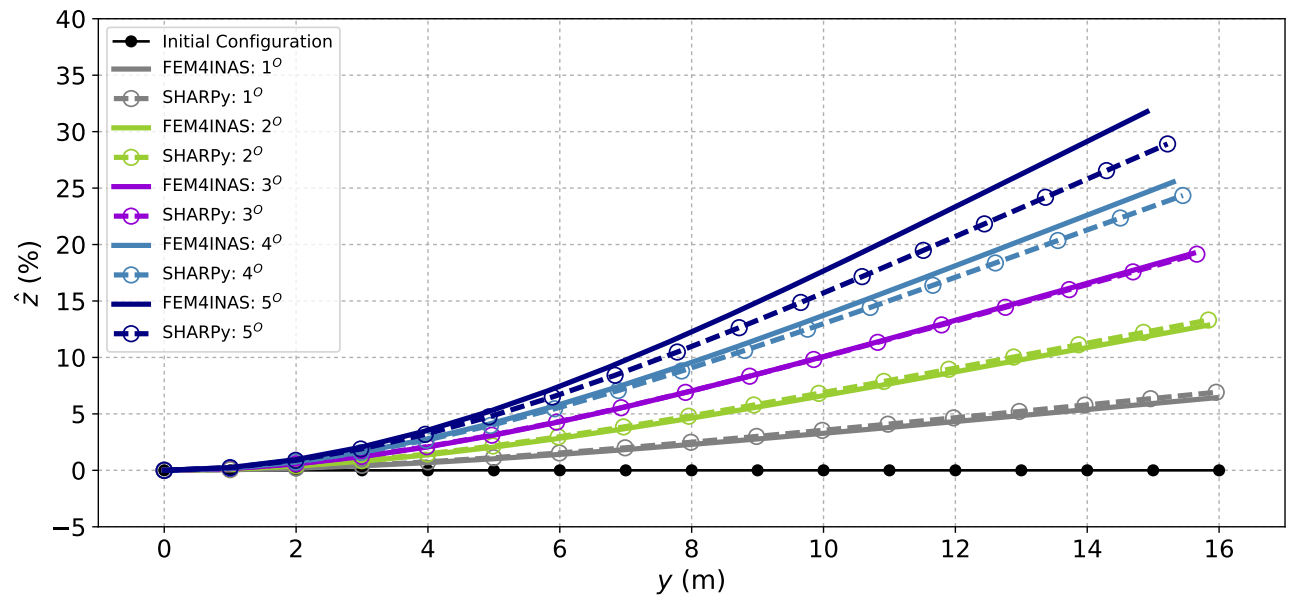

(a) No gravity

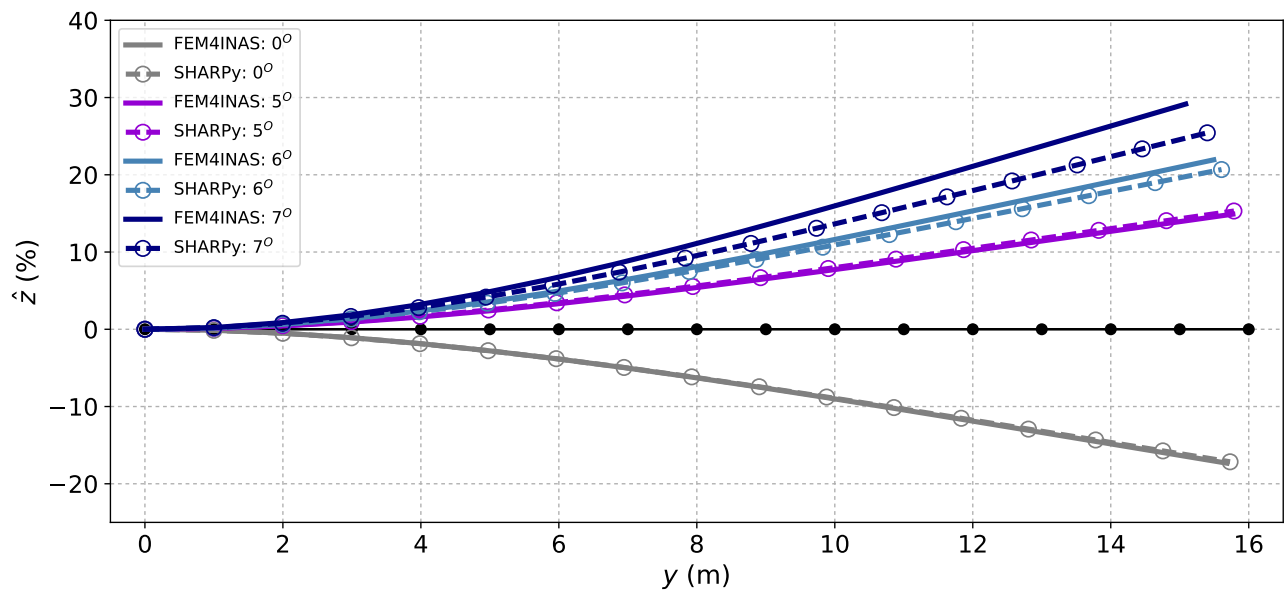

(b) Gravity on

Figure 10: Deformed shape of the wing under fixed angles of attack with and without gravity 
Results indicate the suitability of the proposed method for moderately large deformations, since discrepancies become significant only with deformations over 25\%. For large deformations, the DLM in the initial configuration does not account for the geometricallynonlinear effect in the upwash, thus the module of the follower forces does not decrease as deformations grow larger. On the other hand, a purely linear analysis can be carried out using Eq. (9) around the undeformed configuration and retrieving positions from the expansion of the displacement field: modal coordinates, $\boldsymbol{q}_{\mathbf{0}}$, multiplied by the correspondent mode-shapes, $\boldsymbol{\phi}_{0}$, instead of the nonlinear integration of strains or velocities. The resulting linear equilibrium, however, would miss the shortening effect from the near-inextensionality in the geometrically-nonlinear model, as well as the small inward rotation resulting from follower-force effect in the aerodynamics. In terms of modelling gravity, figure 10(b), it is worth noting that tracking of geometric information allows a very good accuracy in the wing nonlinear deformation under its own weight, even though only the linear initial mass matrix is known to the NMROM, unlike SHARPy, which updates it in every fluid-structure iteration. It is also noticeable that including gravity brings a small additional error to the comparisons, which is due to details in the definition of the spanwise inertia distribution between models.

In order to verify the proposed method for dynamic aeroelasticity, three different forces are applied at the tip of the wing, which is initially in equilibrium at $3^{\circ}$ angle of attack and no gravity. Eq. (10) defines the time history of the forces, which are $\boldsymbol{f}_{1}=[0,0, \pm 25] f(0,0.5,0.5)$, $\boldsymbol{f}_{2}=[0,0, \pm 20] f(0,1,1), \boldsymbol{f}_{3}=[0,0, \pm 15] f(0,2,2)$. The minus sign of the load acts at time zero in the simulation and the positive sign after 10 seconds. Results are shown in Fig. 11, where the response to force $f_{i}$ is identified as $-i$ in the legend. They show a good match between both models, thus demonstrating that the unsteady aerodynamic effects can be appropriately captured by the (linear) DLM as forcing term to the intrinsic equations. The comparison is slightly better for negative loading because deformations decrease and therefore bring both aerodynamic models closer. A lesser difference is seen for the maximum 

effects.

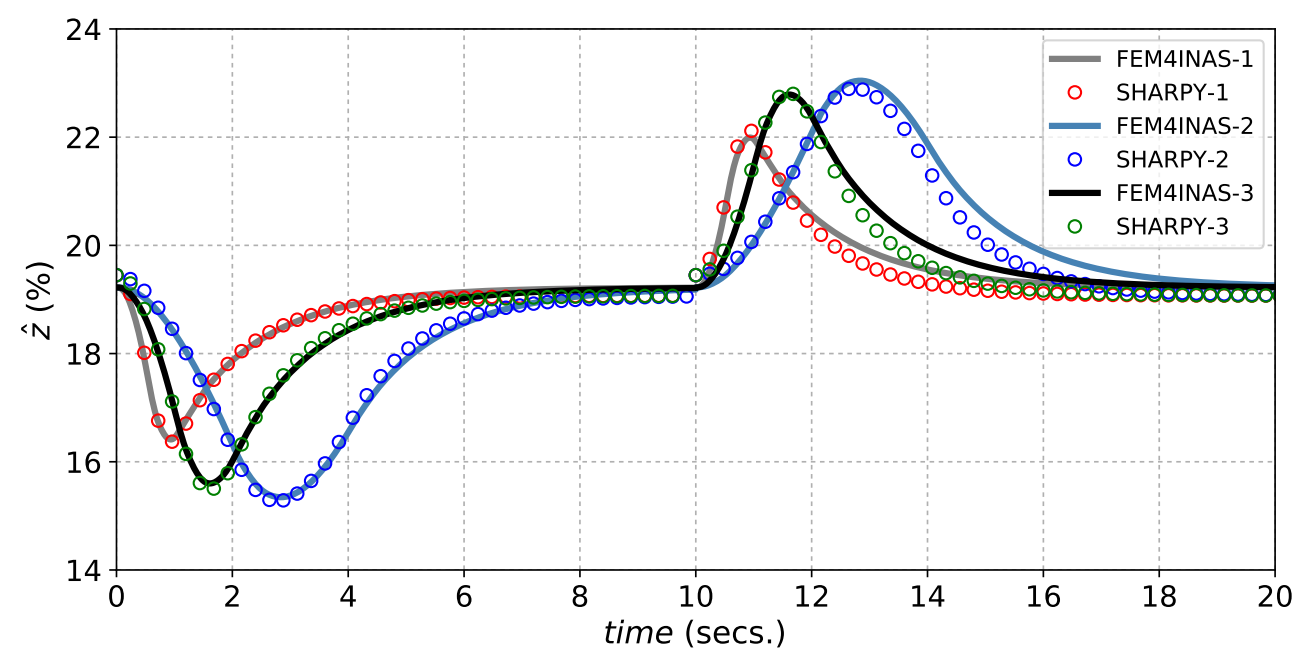

Figure 11: Wing tip vertical displacement for three different doublet-type forces from $3^{\circ}$ static equilibrium

and minimum points of the disturbance, as these are driven mostly by the follower force elfects.

\subsection{Stability and nonlinear response of a cantilever wing-box}

In this final test case, the post-flutter behavior of a wing for which its aeroelastic model is built using linear tools will be considered. The test case is the modified (or heavy) Goland wing with tip stores of Beran et al. (2004), which has been stretched for these results to seek large deformations. A (linear) MSC Nastran model has been built and it is shown in Fig. 12. It is a cantilever wing, initially of $20 \mathrm{ft}$ span, but here increased to $40 \mathrm{ft}$ (that is, $12.129 \mathrm{~m}$, for an aspect ratio of 13.3$)$; it has $4 \mathrm{ft}(1.213 \mathrm{~m})$ structural chord and $6 \mathrm{ft}(1.829$ m) aerodynamic chord. While the geometry is rather simple, it incorporates shell and beam elements to model the skin, ribs and spars, as well as lumped masses. The DLM model has been built with 24 panels in the spanwise direction and 20 panels along the chord. Guyan condensation is employed onto the central points of the ribs. 


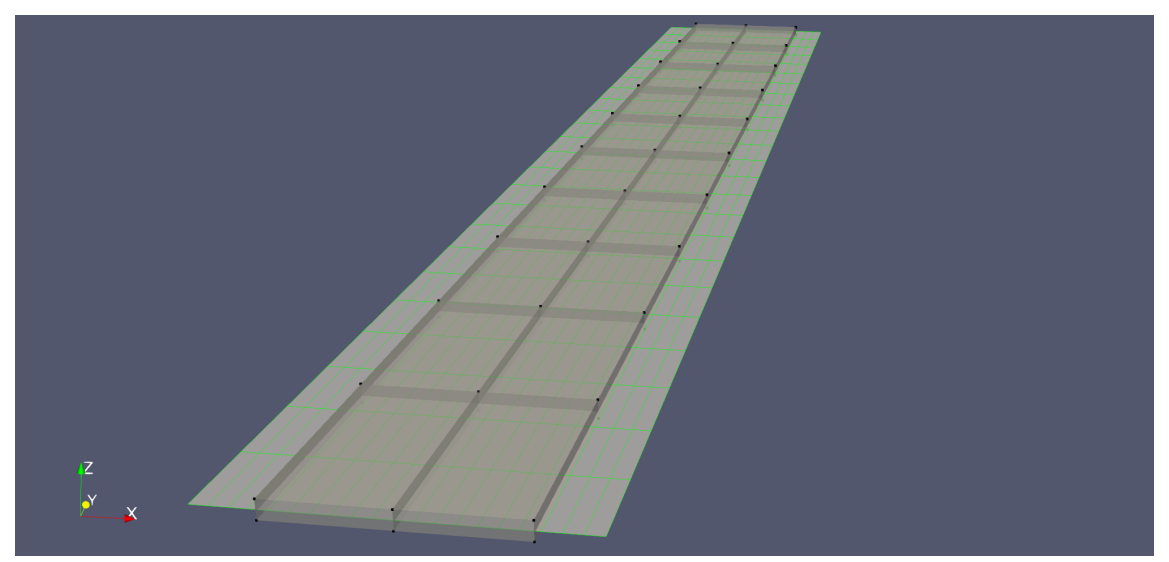

Figure 12: Stretched Goland wing structural and aerodynamic model

\subsubsection{Linear flutter results}

The flutter mechanism for the stretched wing is still driven by the aeroelastic coupling between the first bending and first torsional modes. Therefore, only the first three LNMs are retained for the initial linear analysis. The corresponding AICs, $\boldsymbol{Q}$ (Ma, $k$ ), are calculated for a range of reduced frequencies $k=\left(10^{-9}, 0.01,0.02, \ldots, 1\right)$ for each Mach number of interest. RFAs with 2 and 4 aerodynamic lags are considered. Figure 13 show the imaginary versus real parts of all the components of the $\mathrm{AIC}, \boldsymbol{Q}$, for $\mathrm{Ma}=0.7$ and for the considered range of reduced frequencies (in increments of $\Delta k=0.03$ ). The figure compares the discrete calculations obtained from the DLM, a RFA using Rogers' approximation (equation (5)) and with the poles chosen as in (Castrichini et al., 2016), which is representative of industrial practice, and a second RFA with optimized selection of the aerodynamic lags, as described in section 2.2.1. In Castrichini et al. (2016), the aerodynamic lags are chosen as $\gamma_{0 p}=\frac{k_{\max }}{p}$, while after optimization the 2-pole RFA results in $[1,0.067]$ and the 4-pole in $[1,0.9,0.8,0.096]$ for $\mathrm{Ma}=0.7$. Optimization of pole selection produces a clear improvement on the accuracy of the RFA, which is more marked at higher Mach numbers, where the AICs present higher variations with reduced frequencies. Although the optimized 2-pole approximation may be a good enough fit, 4-poles are used in subsequent analysis.

The flutter speed is next calculated from the eigenvalues of Eq. (9) and compared with 

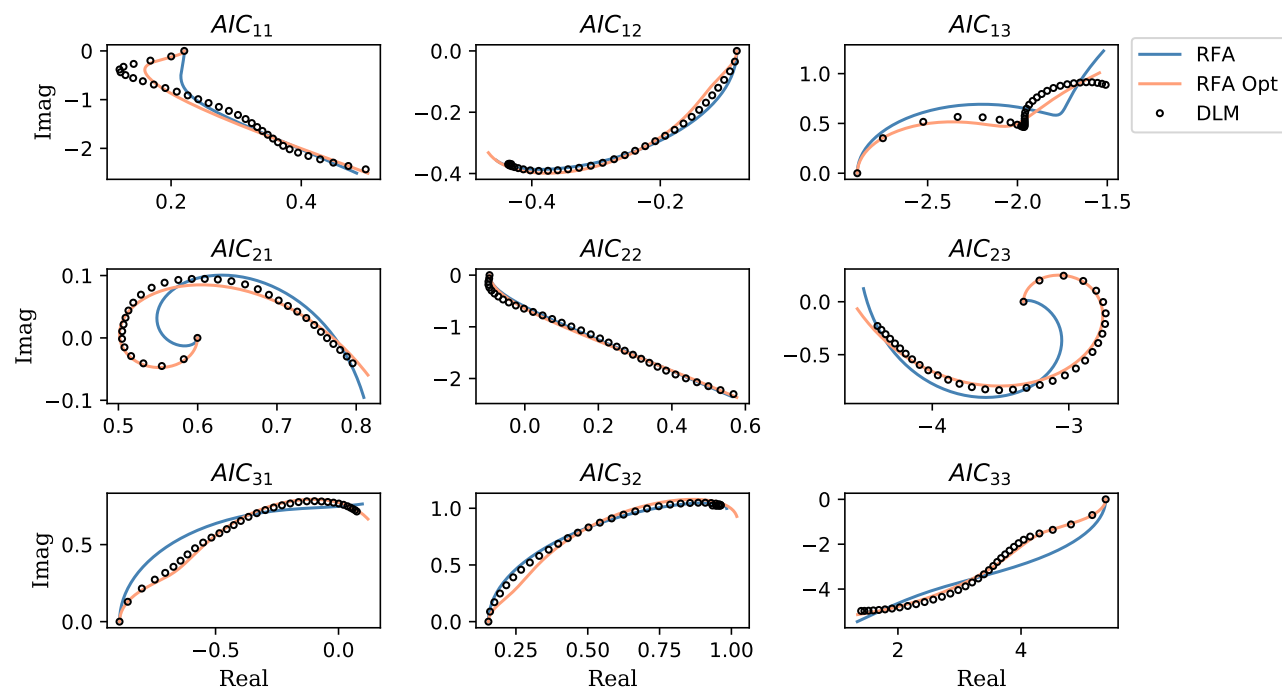

(a) 2-pole approximation
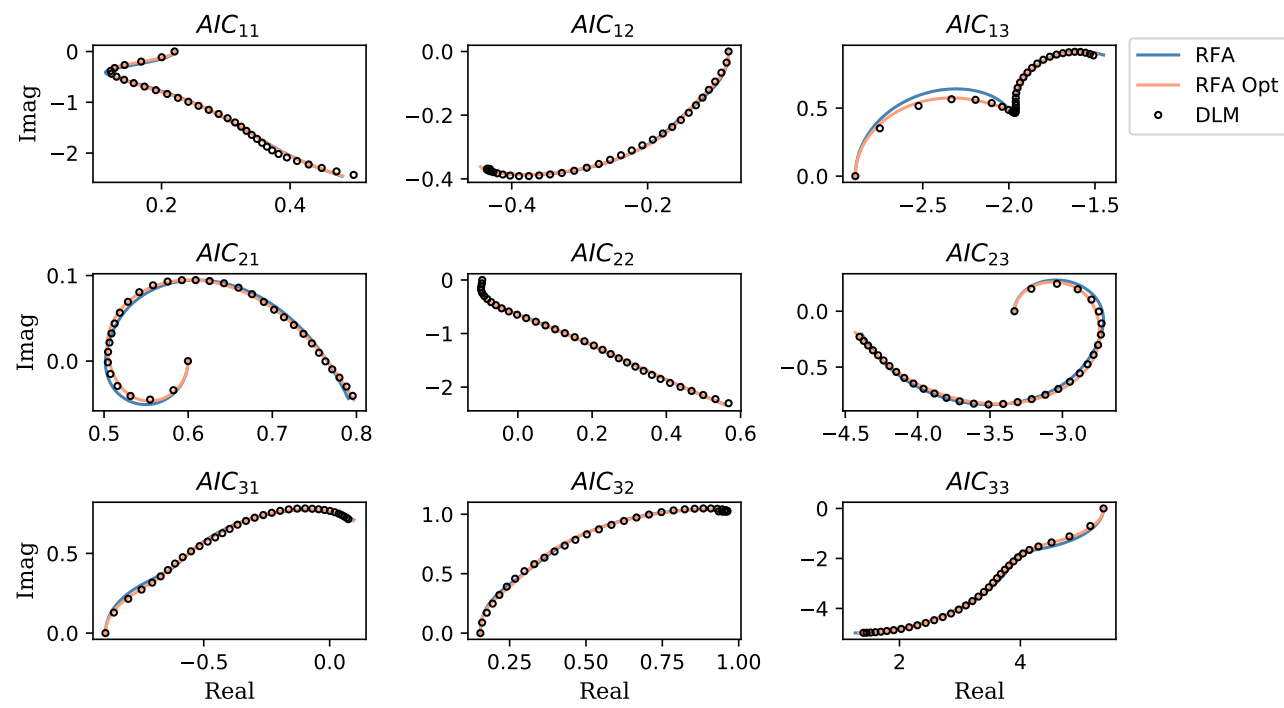

(b) 4-pole approximation

Figure 13: Rational function approximation for $\mathrm{Ma}=0.7$, for equally spaced reduced frequencies in $0 \leq k \leq 1$

the direct frequency-domain results using the PK method in MSC Nastran. Figure 14 shows the flutter speed and frequency for varying Mach numbers, $\mathrm{Ma}=0,0.3 ., 0.5,0.7$ and 0.85. Both full and reduced model results are employed in the MSC Nastran calculations. Stability conditions defined by the eigenvalue analysis of the linearized system (9) are also included, using the 4-lag RFA defined above. As the NMROM reduces to a description in the original 


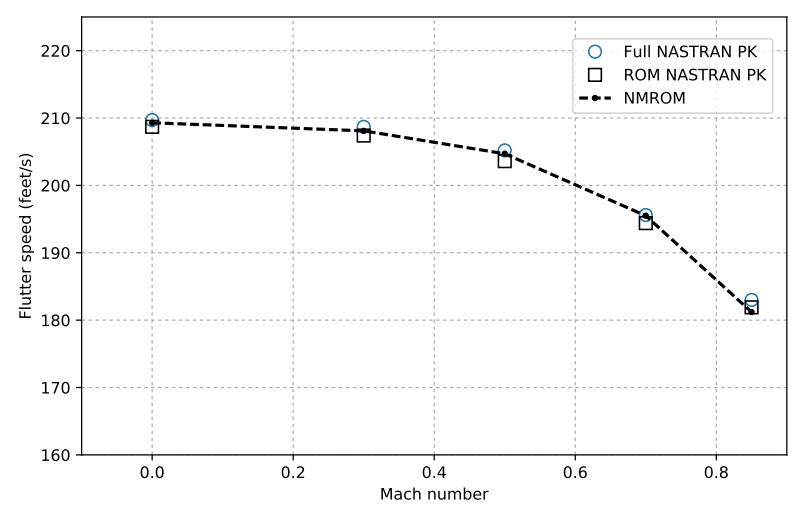

(a) Flutter velocity

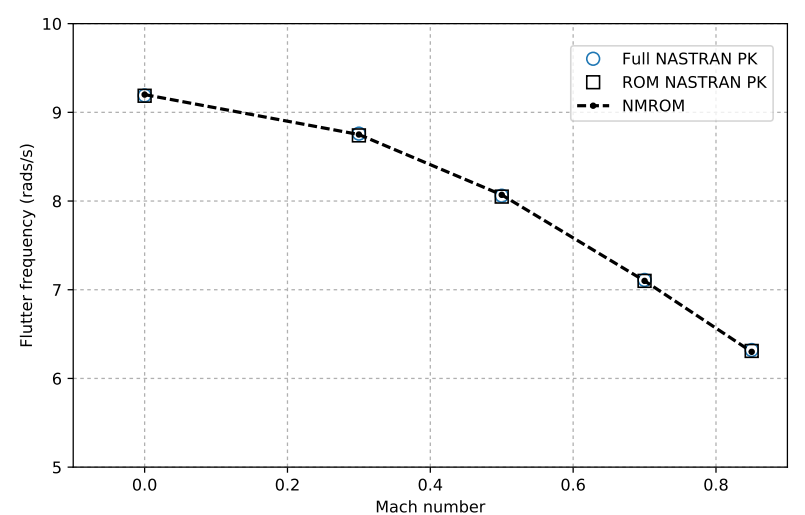

(b) Flutter frequencies

Figure 14: Flutter conditions of the Goland wind for changing Mach number

LNMs under linear assumptions, its flutter boundary is very close to that obtained by MSC Nastran using either the full or the condensed models, with the small differences being driven by the different manipulation of the same AICs between both solution processes. The preservation of the linear model is a fundamental feature of the proposed approach.

\subsubsection{Nonlinear response}

The nonlinear system in Eq. (7) is used to explore the post-flutter behavior of this wing. Twelve modes were employed in the solution, $N_{m}=12$, as this was found to be sufficient to capture the main features on the nonlinear response while keeping computational cost very low. Four aerodynamic lags were again selected, therefore resulting in 48 aerodynamic states. Calculations are carried out at $\mathrm{Ma}=0$, where the flutter equivalent air speed is just below $210 \mathrm{ft} / \mathrm{s}(64.008 \mathrm{~m} / \mathrm{s})$. An initial small perturbation in the form of a parabolic velocity in the $z$-direction is given to initiate the wing dynamics. Figure 15 shows the time history of the vertical tip displacements, normalized by the semi-span, for both linear and nonlinear analysis and equivalent airspeed of $214 \mathrm{ft} / \mathrm{s}(65.227 \mathrm{~m} / \mathrm{s})$, that is, just above the flutter onset. The simulation time is 25 seconds, with time step $\Delta t=0.002$. While both analyses featured a similar initial response, simulations diverged in the linear approach and, on the 


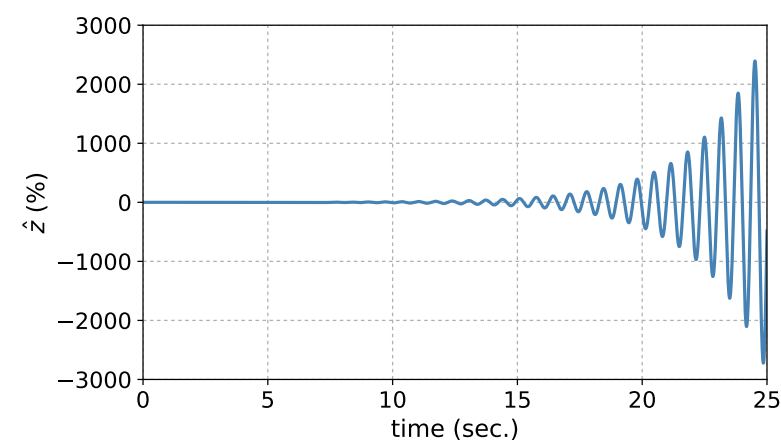

(a) Linear analysis

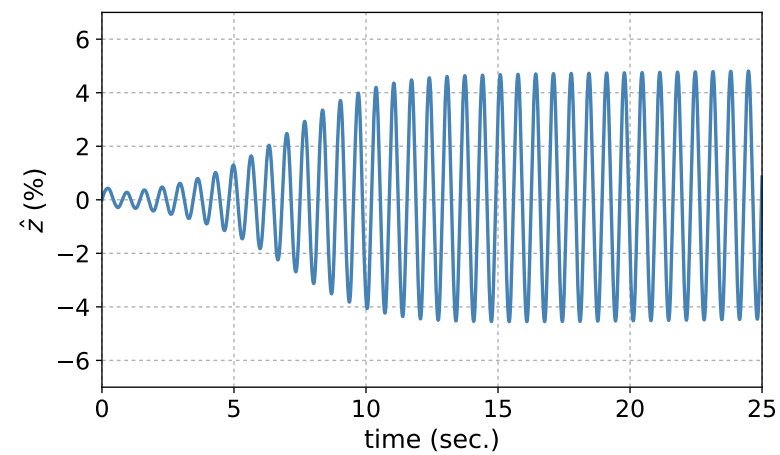

(b) Nonlinear analysis, NMROM

Figure 15: Normalized vertical response of the wing-tip at 0 Mach number and V=214 ft/s the first two velocity modes, $q_{11}$ and $q_{12}$. These modes comprise both out-of-plane bending

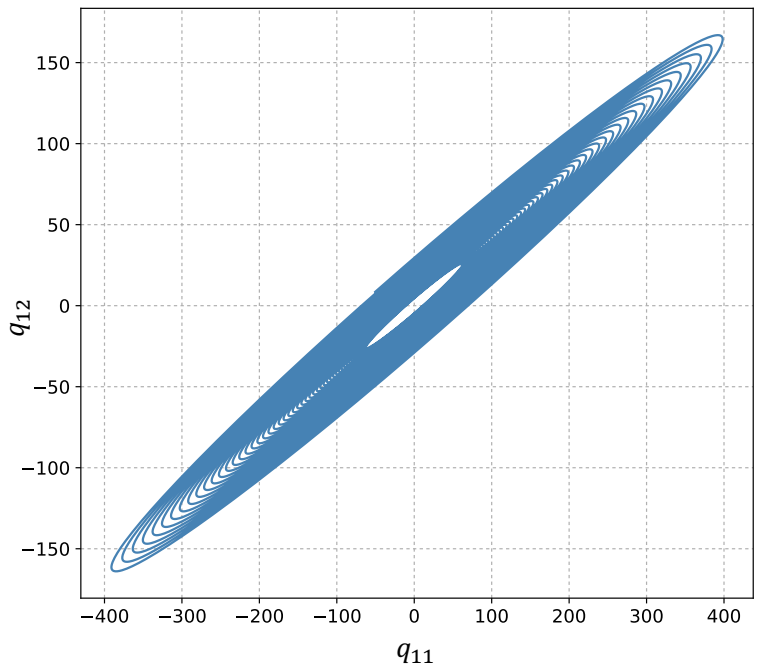

(a) Linear analysis

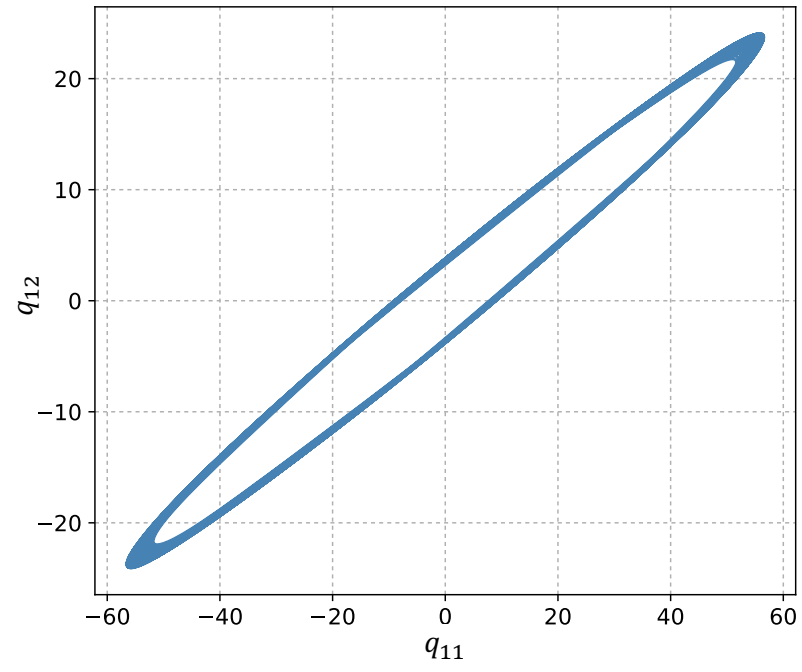

(b) Nonlinear analysis, NMROM

Figure 16: Linear VS nonlinar trajectories of first two modal amplitudes at $214 \mathrm{ft} / \mathrm{s}$

The nonlinear behavior is further explored through the phase plot of the amplitudes of 

et al., 2018).

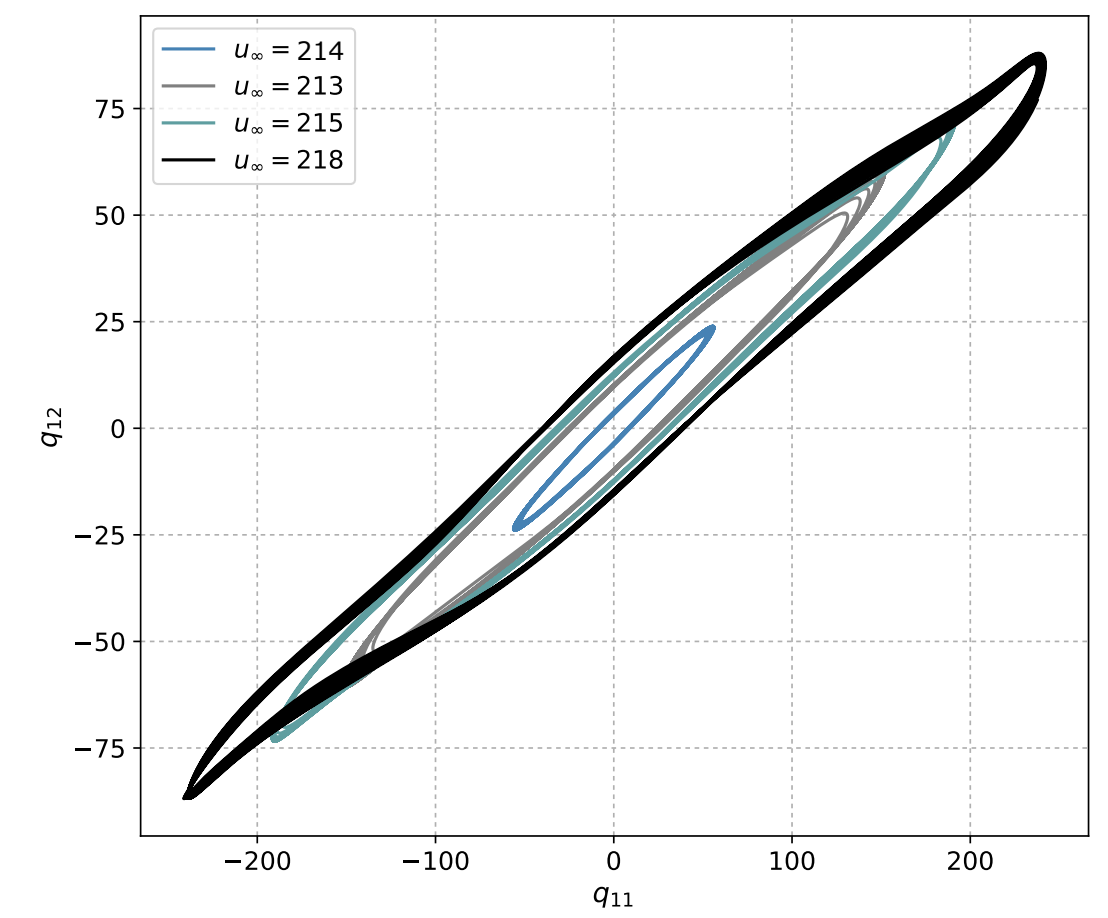

Figure 17: Trajectories of first two modal amplitudes with flow speed from $214 \mathrm{ft} / \mathrm{s}$ to $218 \mathrm{ft} / \mathrm{s}$

$218 \mathrm{ft} / \mathrm{s}$ the shape drawn by the LCO is not an ellipse anymore. This highlights the need for nonlinear approaches even for moderate deformations, as also recently shown in (Farsadi

A key effect in the evolution of the LCOs with larger deformations is the increased nonlinear coupling between modes. In-plane modes are excited through those couplings even though only out-of-plane modes were perturbed in the initial excitation. As an example, Fig. 18 shows the time evolution of the amplitude of the fourth velocity mode, which is a pure in-plane bending mode. Linear and nonlinear results are presented in blue and grey, respectively, and it is clear that the excitation of this mode only takes place in the nonlinear computations. We note however that the in-plane kinematics of the structure do not translate into aerodynamic forces, as they have no effect in the (linearized) aerodynamics of the DLM. Either higher-order panel methods (Kier, 2017), a linearized UVLM (Murua et al., 2014) or a linearized CFD would be needed to account for this effect. 


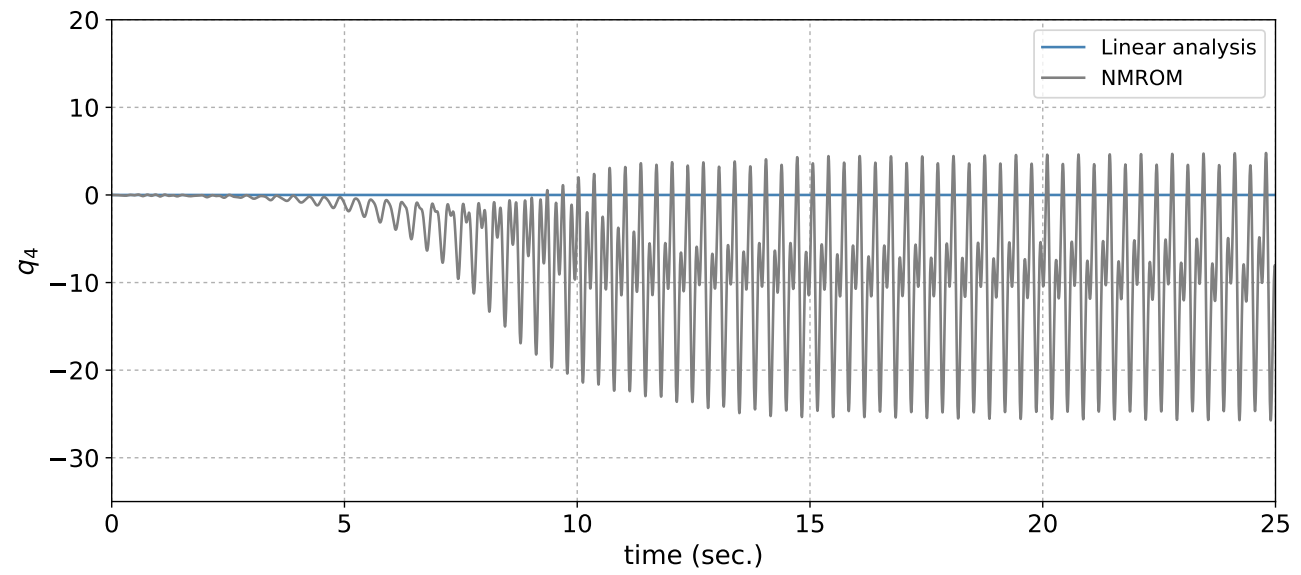

Figure 18: Fourth velocity modal coordinate evolution in time for both linear and nonlinear solutions

\section{Conclusions}

A modal-based description has been presented that seamlessly introduces geometricallynonlinear effects on generic models built for linear dynamic analysis. The proposed approach preserves the linear solution, which is then augmented with the nonlinear effects in beam theory, namely geometric stiffening, follower forces and changes of inertia properties. To achieve this, firstly, a condensed structure along the main load-path of the full 3D model is obtained using techniques of modal condensation. Secondly, the dynamics of the resulting skeleton-like structure are assumed to be driven by the nonlinear beam intrinsic equations, written in modal space. All the parameters in those equations are obtained through closedform expressions involving the condensed stiffness and mass matrices, the linear normal modes and, critically, the coordinates of the grid points. Thirdly, influence coefficient matrices, here obtained from the DLM, describe the unsteady aerodynamics in those modal coordinates. This results in a geometrically-nonlinear yet highly computationally efficient aeroelastic system. The three distinctive features of the proposed approach are that: 1) only linear analysis methods are used to manipulate the original 3D model; 2) the equivalent beam model is directly built in modal space with no need to explicitly identify sectional stiffness and inertia; 3) the problem size can be reduced by selecting the number of modes 
included in the NMROM. Importantly, no model updates are required on either the fluid or the structure (for which only quadratic nonlinearities terms are present), which greatly reduce computational time.

This strategy has been shown to produce excellent results for moderately large deformations with attached flows (over $20 \%$ of the wing span in our simulations). Therefore, the aeroelastic analysis of future transport aircraft with higher aspect ratio wings might find a good compromise of fidelities in this approach. As the computational effort per simulation is modest, this approach would make tractable the thousands of computations required in the design load envelope, to envision control strategies or to run optimized preliminary designs. On-going work is currently extending this methodology to full aircraft aeroelastic simulation process, including trimmed flight calculations, gust responses and control strategies for large deflections.

\section{Acknowledgements}

This research is sponsored by an Industrial Cooperative Award in Science and Technology between the UK Engineering and Physical Sciences Research Council and Airbus Group Innovation.

\section{References}

R. Palacios, C. E. S. Cesnik, E. Y. Reichenbach, Re-Examined Structural Design Procedures for Very Flexible Aircraft, Journal of Aircraft 51 (2014) 1580-1591.

T. E. Noll, S. D. Ishmael, B. Henwood, M. E. Perez-Davis, G. C. Tiffany, J. Madura, M. Gaier, J. M. Brown, T. Wierzbanowski, Technical Findings, Lessons Learned, and Recommendations Resulting from the Helios Prototype Vehicle Mishap, in: NATO/RTO AVT-145 workshop on design concepts, processes and criteria for UAV structural integrity, Florence, Italy. 
M. J. Patil, D. H. Hodges, On the Importance of Aerodynamic and Structural Geometrical Nonlinearities in Aeroelastic Behavior of High-Aspect-Ratio Wings, Journal of Fluids and Structures 19 (2004) 905-915.

S. Qiao, H. Gao, Y. Lyu, L. Hua, F. Wang, Nonlinear Aeroelastic Characteristics Analysis of Composite Wing with High Aspect Ratio Based on Co-Rotational Method, Journal of Fluids and Structures 82 (2018) 619 - 637.

T. M. Kier, An Integrated Model for Lateral Gust Loads Analysis and Dutch Roll Flight Dynamics Using a 3D Panel Method, in: International Forum on Aeroelasticity and Structural Dynamics.

Y. Wang, A. Wynn, R. Palacios, Nonlinear Aeroelastic Control of very Flexible Aircraft Using Model Updating, Journal of Aircraft 55 (2018) 1551-1563.

F. Afonso, J. Vale, É. Oliveira, F. Lau, A. Suleman, A Review on Non-Linear Aeroelasticity of High Aspect-Ratio Wings, Progress in Aerospace Sciences 89 (2017) 40-57.

J. Dizy, R. Palacios, S. Pinho, Homogenisation of Slender Periodic Composite Structures, International Journal of Solids and Structures 50 (2013) 1473-1481.

C. Riso, D. Sanghi, C. E. Cesnik, F. Vetrano, P. Teufel, Parametric Roll Maneuverability Analysis of a High-Aspect-Ratio-Wing Civil Transport Aircraft, in: AIAA Scitech Forum, Orlando, Florida.

R. Palacios, A. Cea, Nonlinear Modal Condensation of Large Finite-Element Models: An Application of Hodges' Intrinsic Theory, AIAA Journal 57 (2019) 4255-4268.

R. R. Medeiros, C. E. S. Cesnik, E. B. Coetzee, Computational Aeroelasticity Using ModalBased Structural Nonlinear Analysis, AIAA Journal 58 (2020) 362-371. 
M. P. Mignolet, A. Przekop, S. A. Rizzi, S. M. Spottswood, A Review of Indirect/NonIntrusive Reduced Order Modeling of Nonlinear Geometric Structures, Journal of Sound and Vibration 332 (2013) 2437-2460.

Y. Wang, R. Palacios, A. Wynn, A Method for Normal-Mode-Based Model Reduction in Nonlinear Dynamics of Slender Structures, Computers and Structures 159 (2015) 26-40.

D. H. Hodges, Geometrically Exact, Intrinsic Theory for Dynamics of Curved and Twisted Anisotropic Beams, AIAA Journal 41 (2003) 1131-1137.

A. H. Modaress-Aval, F. Bakhtiari-Nejad, E. H. Dowell, D. A. Peters, H. Shahverdi, A Comparative Study of Nonlinear Aeroelastic Models for High Aspect Ratio Wings, Journal of Fluids and Structures 85 (2019) 249-274.

T. Kim, Flutter Prediction Methodology Based on Dynamic Eigen Decomposition and Frequency-Domain Stability, Journal of Fluids and Structures 86 (2019) $354-367$.

J. Murua, R. Palacios, J. Michael, R. Graham, Applications of the Unsteady Vortex-Lattice Method in Aircraft Aeroelasticity and Flight Dynamics, Progress in Aerospace Sciences 55 (2012) 46-72.

H. Güner, D. Thomas, G. Dimitriadis, V. E. Terrapon, Unsteady Aerodynamic Modeling Methodology Based on Dynamic Mode Interpolation for Transonic Flutter Calculations, Journal of Fluids and Structures 84 (2019) 218-232.

R. Palacios, Invariant Manifolds in Beam Dynamics: Free Vibrations and Nonlinear Normal Modes, in: Encyclopedia of Continuum Mechanics, Springer Berlin Heidelberg, 2017, pp. $1-8$.

Z.-Q. Qu, Model Order Reduction Techniques: With Applications in Finite Element Analysis, Springer, USA, 1st edition, 2010. 
R. J. Guyan, Reduction of Stiffness and Mass Matrices, AIAA Journal 3 (1965) 380-380.

W. P. Rodden, Theoretical and Computational Aeroelasticity, Crest Publishing, California, USA, 2011.

M. Karpel, Design for Active Flutter Suppression and Gust Alleviation Using State-Space Aeroelastic Modeling, Journal of Aircraft 19 (1982) 221-227.

R. M. Botez, D. E. Biskri, Unsteady Aerodynamic Forces Mixed Method for Aeroservoelasticity Studies on an F/A-18 Aircraft, Journal of Aircraft 44 (2007) 1378-1383.

A. D. Dinu, R. M. Botez, I. Cotoi, Aerodynamic Forces Approximations Using the Chebyshev Method for Closed-Loop Aeroservoelasticity Studies, Canadian Aeronautics and Space Journal 51 (2005) 167-175.

K. L. Roger, Airplane Math Modeling Methods for Active Control Design, Structural Aspects of Active Controls, AGARD CP-228 (1977) 4.1-4.11.

M. L. Baker, P. J. Goggin, B. A. Winther, DTIC ADP010476: Aeroservoelastic Modeling, Analysis, and Design Techniques for Transport Aircraft, RTO MP-36: Structural Aspects of Flexible Aircraft Control (1999).

S. H. Tiffany, W. M. Adams, Nonlinear Programming - Extensions to Rational Function Approximation Methods for Unsteady Aerodynamic Forces Nonlinear Programming Extensions to Rational Function Approximation Methods for Unsteady Aerodynamic Forces, NASA Technical Paper (1988). NASA-TP-2776.

W. Eversman, A. Tewari, Consistent Rational-Function Approximation for Unsteady Aerodynamics, Journal of Aircraft 28 (1991) 545-552.

Y. Wang, A. Wynn, R. Palacios, Nonlinear Modal Aeroservoelastic Analysis Framework for Flexible Aircraft, AIAA Journal 54 (2016) 1-16. 
M. J. Patil, D. H. Hodges, C. E. Cesnik, Limit-Cycle Oscillations in High-Aspect-Ratio Wings , Journal of Fluids and Structures 15 (2001) 107-132.

A. del Carre, A. Muñoz-Simón, N. Goizueta, R. Palacios, SHARPy: A Dynamic Aeroelastic Simulation Toolbox for very Flexible Aircraft and Wind Turbines, Journal of Open Source Software 4 (2019) 1885.

P. S. Beran, N. S. Khot, F. E. Eastep, R. D. Snyder, J. V. Zweber, Numerical Analysis of Store-Induced Limit-Cycle Oscillation, Journal of Aircraft 41 (2004) 1315-1326.

A. Castrichini, V. Hodigere Siddaramaiah, D. E. Calderon, J. E. Cooper, T. Wilson, Y. Lemmens, Nonlinear Folding Wing Tips for Gust Loads Alleviation, Journal of Aircraft 53 (2016) 1391-1399.

T. Farsadi, M. Rahmanian, A. Kayran, Geometrically Nonlinear Aeroelastic Behavior of Pretwisted Composite Wings Modeled as Thin Walled Beams, Journal of Fluids and Structures 83 (2018) 259-292.

J. Murua, P. Martínez, H. Climent, L. van Zyl, R. Palacios, T-Tail Flutter: Potential-Flow Modelling, Experimental Validation and Flight Tests, Progress in Aerospace Sciences 71 (2014) 54-84. 